\title{
BIBLIOGRAFÍA DE DERECHO INTERNACIONAL PRIVADO (2019)
}

\section{PRIVATE INTERNATIONAL LAW BIBLIOGRAPHY (2019)}

\author{
Alfonso-Luis Calvo Caravaca \\ Catedrático de Derecho internacional privado \\ Universidad Carlos III de Madrid \\ ORCID ID: 0000-0003-2236-4641 \\ JaVier CARrascosa GonzÁlez \\ Catedrático de Derecho internacional privado \\ Universidad de Murcia \\ ORCID ID: 0000-0002-0347-7985
}

DOI: https://doi.org/10.20318/cdt.2021.6282

\section{Obras generales}

M. Bogdan/M. Pertegás Sender, Concise introduction to EU private international law, $4^{\mathrm{a}}$ ed., Amsterdam, Europa Law Publishing, 2019.

A. BRIGGS, The conflict of laws, $4^{\mathrm{a}}$ ed., Oxford, Oxford University Press, 2019.

E. Brödermann/J. Rosengarten, Internationales Privat- und Zivilverfahrensrecht: Anleitung zur systematischen Fallbearbeitung (einschließlich schiedsrechtlicher Fälle), $8^{\mathrm{a}}$ ed., München, Verlag Franz Vahlen, 2019.

A.-L. Calvo Caravaca/J. Carrascosa González (Dir.), Compendio de Derecho internacional privado, Murcia, Rapid Centro Color, 2019.

F. CASSANDRO, Compendio di diritto internazionale privato e processuale: aggiornato con tutte le ultime novità normative e giurisprudenziali, $3^{\mathrm{a}}$ ed., Piacenza, La Tribuna, 2019.

G. Christand, Handbuch Italienisches Internationales Privatrecht, Wien, LexisNexis, München, C.H. Beck, 2019.

J. Clavel-Thoraval, Les indispensables du droit international privé, Paris, Ellipses, 2019.

A. Furrer/D. Girsberger/M. Müller-Chen/D. Schramm, Internationales Privatrecht, $4^{a}$ ed., Zürich, Basel, Genf, Schulthess, 2019.

V.C. Govindaras, The conflict of laws in India: inter-territorial and inter-personal conflict, $2^{\mathrm{a}}$ ed., New Delhi, Oxford University Press, 2019.

P. Hay, Conflict of laws, $8^{\mathrm{a}}$ ed., St. Paul, MN, West Academic Publishing, 2019.

B. von Hoffmann/K. Thorn, Internationales Privatrecht: einschließlich der Grundzüge des Internationalen Zivilverfahrensrechts, 10 $0^{\mathrm{a}}$ ed., München, C.H. Beck, 2019.

R. Hüsstege/H.-P. Mansel (Hrsg.), Rom-Verordnungen: Rom I (Vertragliche Schuldverhältnisse), Rom II (Außervertragliche Schuldverhältnisse), Rom III (Ehescheidung, Trennung), EuGüVO/EuPartVO (Eheliches Güterrecht/lebenspartnerschaftliches Güterrecht), HUP (Haager Unterhaltsprotokoll), EuErbVO (Erbrecht), $3^{\mathrm{a}}$ ed., Baden-Baden, Nomos, 2019.

R. JAFFERALI/N. Watté, Règles générales du droit international privé belge et européen, Bruxelles, Larcier, 2019.

A. JunKer, Internationales Privatrecht, $3^{\text {a }}$ ed., München, C.H. Beck, 2019. 
A. JunkER, Internationales Zivilprozessrecht, $4^{\mathrm{a}}$ ed., München, C.H. Beck, 2019.

K. KReBS, Internationales Privatrecht, $3^{\mathrm{a}}$ ed., Heidelberg, C.F. Müller, 2019.

J. Kren KostKIEwICZ et AL., IPRG/LugÜ: Kommentar: Bundesgesetz über das Internationale Privatrecht, Lugano-Übereinkommen und weitere Erlasse, 2 ${ }^{\mathrm{a}}$ ed., Zürich, Orell Füssli Verlag, 2019.

L. Levita/A. BIfulco/C. Iorio/A.V. SARnataro, Compendio di diritto internazionale privato e processuale, Roma, Dike giuridica editrice, 2019.

P. Mankowski, Internationales Privatrecht, vol. 2 (Besonderer Teil), $2^{\mathrm{a}}$ ed., München, C.H.Beck, 2019.

H.-P. Mansel, Internationales Privatrecht, Baden-Baden, Nomos, 2021.

P. MaYer/V. HeuzÉ/B. Remy, Droit international privé, 12a ed., Issy-les-Moulineaux, LGDJ, 2019.

V. De Oliveira Mazzuoli, Curso de direito internacional privado, $4^{\mathrm{a}}$ ed., Rio de Janeiro, Editora Forense, 2019.

R. Mortensen/R. Garnett/M. Keyes, Private international law in Australia, $4^{\mathrm{a}}$ ed., Chatswood, NSW, LexisNexis Butterworths Australia, 2019.

A. Pasquariello, Compendio di diritto internazionale privato e processuale, $8^{\mathrm{a}}$ ed., Molfetta, Neldiritto editore, 2019.

B.W. RechsteIner, Direito internacional privado: teoria e prática, 20ª ed., São Paulo, Saraiva, 2019.

S.C. Symeonides/W.C. Perdue, Conflict of laws: American, comparative, international: cases and materials, $4^{\text {a }}$ ed., St. Paul, MN, West Academic Publishing, 2019.

N. WATtÉ/R. JAFFERALI, Règles générales du droit international privé belge et européen, Bruxelles, Larcier Legal, 2019.

\section{El Derecho internacional privado}

E. Avato/M.M. WinKLER, "Reinforcing the public law taboo: a note on Hellenic Republic v Nikiforidis", European Current Law Yearbook, 2019, pp. 15-27.

S. BARIATTI, "Volontà delle parti e internazionalità del rapporto giuridico: alcuni sviluppi recenti nella giurisprudenza della Corte di giustizia sui regolamenti europei in materia di diritto internazionale privato", RDIPP, LV, 3, 2019, pp. 513-534.

G. CAVALIER, "Règle fiscale française, droit international privé (communautaire) et droit (non fiscal) étranger: quelle méthode d'articulation?", Revue internationale des services financiers, $\mathrm{n}^{\circ}$ 1, 2019, pp. 82-91.

A. Cebrián Salvat/I. Lorente Martínez (Dir.), Innovación docente y Derecho internacional privado, Granada, Editorial Comares, 2019.

A. DAvì, "Il riconoscimento delle situazioni giuridiche costituite all'estero nella prospettiva di una riforma del sistema italiano di diritto internazionale privato", RDI, CII, 2, 2019, pp. 319-419.

C. Devaux, La fabrique du droit du commerce international: réguler les risques de capture, Bruxelles, Bruylant, 2019.

C. KoHLer, "Gelebte Rechtsvergleichung am EuGH: europäisches Kollisionsrecht in Theorie und Praxis", ZEuP, 27, 2, 2019, pp. 337-357.

J. Meeusen, "Comparing interstate and European conflict of laws: from a constitutional perspective can the United States inspire the European Union?", AJCL, 67, 3, 2019, pp. 637-676.

G. SCHULze, "Individuelle und überindividuelle Interessen im IPR", en Die Person im internationalen Privatrecht: Liber Amicorum Erik Jayme, Tübingen, Mohr Siebeck, 2019, pp. 183-206.

S.C. Symeonides, The "private" in private international law, The Hague, Eleven International Publishing, 2019.

\section{El Derecho internacional privado: Fuentes}

American Law Institute, Restatement of the law, third. Conflict of laws: Preliminary draft $n^{\circ} 5$ (October 23, 2019), St. Paul, MN, American Law Institute, 2019. 
S. ARNOLD/S. ZwirLeIN-ForsChNER, "Die Entwicklung der Rechtsprechung zum europäischen internationalen Privatrecht", GPR, 16, 6, 2019, pp. 262-277.

L. D’Avout/S. Huber, “Droit international privé de l'Union européenne (2018)", JDI Clunet, 146, 4, 2019, pp. 1413-1509.

R.J. BADER, Koordinationsmethoden im internationalen Privat- und Verfahrensrecht, Tübingen, Mohr Siebeck, 2019.

S. BARIATTI, "Volontà delle parti e internazionalità del rapporto giuridico: alcuni sviluppi recenti nella giurisprudenza della Corte di giustizia sui regolamenti europei in materia di diritto internazionale privato", RDIPP, LV, 3, 2019, pp. 513-534.

M. BHATTI, Islamic law and international commercial arbitration: The application of shari 'a in contemporary international commercial arbitration, London, New York, Routledge, 2019.

C. BRIĖRE, "Chronique de droit international privé 2019: suite et fin", Petites affiches, 408, n 238, 2019, pp. 24-31.

A. BRIGGS, "Brexit and private international law: an English perspective", RDIPP, LV, 2, 2019, pp. 261283.

A.-L. Calvo Caravaca/J. Carrascosa González (Dir.), El Tribunal Supremo y el Derecho internacional privado, 2 vols., Murcia, Rapid Centro Color, 2019.

A. Di Stasi, Tutela dei diritti fondamentali e spazio europeo di giustizia, Napoli, Editoriale scientifica, 2019.

V. ÉGÉA/E. GuINCHARD/G. PAYAN, "Espace judiciaire européen en matière civile”, Revue trimestrielle de droit européen, $\mathrm{n}^{\circ} 3,2019$, pp. 759-769.

D. EHLERS, "Grundrechtsbindung und Grundrechtsschutz von Unternehmen im deutschen und europäischen Recht", DVBl., 134, 7, 2019, pp. 397-406.

M. GernerT, "Harter Brexit und IPR: vorbereitende Papiere für einen ungeregelten Austritt des Vereinigten Königreichs”, IPRax, 39, 4, 2019, pp. 365-369.

M. HABERSACK/M. EHRL, "Verantwortlichkeit inländischer Unternehmen für Menschenrechtsverletzungen durch ausländische Zulieferer: de lege lata und de lege ferenda", $A c P, 219,2$, 2019, pp. 155-210.

W.J. HAU, "Europeanisation of civile procedure: overcoming follow-up fragmentation through bottom-up harmonisation?", en Civil procedure and harmonisation of law, 2019, pp. 61-75.

M. Heidemann, Transnational Commercial Law, London, Macmillan International Higher Education, Red Globe Press, 2019.

J. von HeIn/E.-M. KIEnINGer/G. RüHL (Eds.), How European is European private international law?: Sources, court practice, academic discourse, Cambridge, Intersentia, 2019.

I.S. JARASS, Privates Einheitsrecht, Tübingen, Mohr Siebeck, 2019.

W.J. Kassir, Autonomie de la volonté et contrat sans loi en droit international privé des contrats: consécration mondiale, risques d'exacerbation et nécessité de préserver l'autorité de la loi dans les contrats internationaux, Beyrouth, Point Delta, Issy-les-Moulineaux, LGDJ, 2019.

C. Kohler, "Gelebte Rechtsvergleichung am EuGH: europäisches Kollisionsrecht in Theorie und Praxis", ZEuP, 27, 2, 2019, pp. 337-357.

X.E. KRAMER, "A common discourse in European private international law?: a view from the Court system", en How European is European private international law?, 2019, pp. 215-234.

H.-P. Mansel/K. Thorn/R. Wagner, "Europäisches Kollisionsrecht 2018: Endspurt!”, IPRax, 39, 2, 2019, pp. 85-119.

J. Meeusen, "Comparing interstate and European conflict of laws: from a constitutional perspective can the United States inspire the European Union?", AJCL, 67, 3, 2019, pp. 637-676.

A. Nylund/M. STRANDBerg, Civil procedure and harmonisation of law: the dynamics of EU and international treaties, Cambridge, Intersentia, 2019.

J.J. Pérez Milla, El espacio del Derecho interregional tras los reglamentos de la Unión Europea sobre familia y sucesiones mortis causa, Zaragoza, El Justicia de Aragón, 2019.

H. SAUER, "Europarechtliche Schranken internationaler Gerichte: eine Rekonstruktion der Autonomie des Unionsrechts anlässlich des CETA-Gutachtens des EuGH”, JZ, 74, 19, 2019, pp. 925-935. 
A. Staudinger, "10 Jahre Rom II-VO und Haager Straßenverkehrsübereinkommen: Zeit für eine Bestandsanalyse im Lichte des Brexit und der angedachten EU-Erweiterung", Deutsches Autorecht, 89, 12, 2019, pp. 669-675B. SUJECKI, "Entwicklung des europäischen Privat- und Zivilverfahrensrechts in den Jahren 2017 und 2018", EWS, 30, 6, 2019, pp. 315-322.

E. TretTHAhN-Wolski/A. FöRstel, "Der Brexit von Rom und Brüssel: zu den Auswirkungen des Austritts des Vereinigten Königreichs aus der EU auf das internationale Zivil- und Zivilprozessrecht”, ÖJZ, $74,11,2019$, pp. 485-490.

C.E. Tuo, "The consequences of Brexit for recognition and enforcement of judgments in civil and commercial matters: some remarks", RDIPP, LV, 2, 2019, pp. 302-308.

C. Voss, Die Durchbrechung der Rechtskraft nationaler Zivilgerichtsurteile zu Gunsten des unionsrechtlichen "effet utile"?: die Reichweite der "Klausner Holz"-Entscheidung des Europäischen Gerichtshofs und ihre Auswirkungen auf das deutsche Zivilprozessrecht, Baden-Baden, Nomos, 2019.

R. WAGNER, "Zwanzig Jahre justizielle Zusammenarbeit in Zivilsachen", IPRax, 39, 3, 2019, pp. 185-200.

C. WARIN, "Individual rights and collective interests in EU law: three approaches to a still volatile relationship", CMLR, 56, 2, 2019, pp. 463-487.

C. WendelsteIn, “'Menschenrechtliche' Verhaltenspflichten im System des internationalen Privatrechts", RabelsZ, 83, 1, 2019, pp. 111-153.

F.M. WILKE, A conceptual analysis of European private international law: the general issues in the EU and its member states, Cambridge, Intersentia, 2019.

\section{Competencia judicial internacional}

S. Arnold/T. Garber, "Ein vermeintlicher Pyrrhussieg für Griechenland: die Grenzen staatlicher Souveränität im internationalen Zivilverfahrensrecht (zu EuGH, 15.11.2018, C-308/17, Hellenische Republik vs. Kuhn, unten S. 417, Nr. 37, und öOGH, 22.1.2019, 10 Ob 103/18x, unten S. 419, Nr. 38)", IPRax, 39, 5, 2019, pp. 385-391.

A. Arzandeh, Forum (non) conveniens in England: past, present, and future, Oxford, Hart, 2019.

S. ClaVEL, "Protection juridictionnelle effective et règles de droit international privé", JDI Clunet, 146, 3, 2019, pp. 695-716.

C. KLEINER, "Compétence judiciaire: CJUE, 1re ch., 15 nov. 2018, aff. C-308/17, Hellenische Republik c/ Leo Kuhn”, JDI Clunet, 146, 3, 2019, pp. 854-867.

M. La MANNA, "The ECTHR Grand Chamber's judgment in the Naiit-Liman case: an unnecessary clarification of the reach of forum necessitatis jurisdiction?", RDIPP, LV, 2, 2019, pp. 349-382.

P. Mankowski, "Griechische Staatsanleihen und der griechische Schuldenschnitt vor dem EuGH: zugleich Besprechung EuGH v. 15.11.2018, Rs. C-308/17, ZIP 2018, 2290, Kuhn, (Folge Zwei)”, ZIP, 40, 5, 2019, pp. 193-202.

\section{Derecho aplicable (I). Técnicas de reglamentación. La norma de conflicto}

R.J. BADER, Koordinationsmethoden im internationalen Privat- und Verfahrensrecht, Tübingen, Mohr Siebeck, 2019.

S. BIEHL, Eingriffsnormen und Schiedsvereinbarungen: eine Untersuchung der Auswirkungen von Eingriffsnormen auf die Anerkennung einer internationalen Schiedsvereinbarung in der Einredesituation, Berlin, Peter Lang, 2019.

A. DAvì, "Il riconoscimento delle situazioni giuridiche costituite all'estero nella prospettiva di una riforma del sistema italiano di diritto internazionale privato", RDI, CII, 2, 2019, pp. 319-419.

M. Françoise, "L'exception et la règle de conflit européen: l'exception d'ordre public et la loi de police: deux exceptions régulées au service de la norme européenne", en L'exception en droit de l'Union européenne, 2019, pp. 301-312.

C. Harms, Neuauflage der Datumtheorie im Internationalen Privatrecht, Tübingen, Mohr Siebeck, 2019. 
A. HeMLER, Die Methodik der "Eingriffsnorm" im modernen Kollisionsrecht: zugleich ein Beitrag zum internationalen öffentlichen Recht und zur Natur des ordre public, Tübingen, Mohr Siebeck, 2019.

M. Ho-DAC, "L'arrêt da Silva Martins de la Cour de justice de l'Union européenne, expression des 'rapports de méthodes' dans l'ordre juridique européen", Revue trimestrielle de droit européen, $\mathrm{n}^{\circ} 4$, 2019, pp. 869-882.

P. Mankowski, "Politik und missliebige drittstaatliche Eingriffsnormen: der Fall Kuwait Airways: zugleich Besprechung zu OLG Frankfurt, RIW 2019, 231”, RIW, 65, 4, 2019, pp. 180-184.

H.-P. Mansel, "Methoden des internationalen Privatrechts: Personalstatut, Verweisung und Anerkennung", en Die Person im internationalen Privatrecht: Liber Amicorum Erik Jayme, Tübingen, Mohr Siebeck, 2019, pp. 27-46.

H. Muir WatT, Discours sur les méthodes du droit international privé (des formes juridiques de l'interaltérité): cours général de droit international privé, Leiden, Brill Nijhoff, 2019.

S. SchwAmberger/L. KLever, "Sammelklage europäischer Prägung?", Wirtschaftsrechtliche Blätter, 33, 1, 2019, pp. 12-20.

T. SzaBADos, Economic sanctions in EU private international law, Oxford, Hart, 2019.

M. Tнол, "Forumfremde Eingriffsnormen und Außenpolitik im IPR: die Israel-Boykottgesetzgebung arabischer Staaten und ihre Berücksichtigung vor deutschen Gerichten (zu OLG Frankfurt a.M., 25.9.2018, 16 U 209/17, unten S. 321, Nr. 30)", IPRax, 39, 4, 2019, pp. 301-306.

\section{Derecho aplicable (II). Aplicación del Derecho extranjero}

M. BENECKE, Gesetzesumgehung im Zivilrecht: Lehre und praktischer Fall im allgemeinen und internationalen Privatrecht, Tübingen, Mohr Siebeck, 2019.

C. Benicke, "Anpassungsproblematik beim Zusammentreffen deutschen Erbrechts mit englischem Eltern-Kind-Statut (zu OLG München, 10.2.2017, 34 Wx 175/16, unten S. 162, Nr. 16)”, IPRax, 39, 2, 2019, pp. 132-140.

B. CENTNER, Iura novit curia in internationalen Schiedsverfahren: eine historisch-rechtsvergleichende Studie zu den Grundlagen der Rechtsermittlung, Tübingen, Mohr Siebeck, 2019.

H. DitTMERs, Die Anpassung im europäischen Internationalen Privatrecht, Berlin, Peter Lang, 2019.

S. Fragoso, Ordem pública no Mercosul e na União Europeia: critérios de aplicação, Curitiba, Juruá Editora, 2019.

M. FrançoIse, "L'exception et la règle de conflit européen: l'exception d'ordre public et la loi de police: deux exceptions régulées au service de la norme européenne", en L'exception en droit de l'Union européenne, 2019, pp. 301-312.

O. Meyer, "Parteiautonomie bei Mehrrechtsstaaten", RabelsZ, 83, 4, 2019, pp. 721-759.

L. RADEMACHER, "Favor laesi und renvoi: Verweisungsart bei Distanzdelikten (zu OLG Hamm, 3.5.2017, 3 U 30/17, unten S. 165, Nr. 17)", IPRax, 39, 2, 2019, pp. 140-147.

\section{Eficacia extraterritorial de decisiones}

G. CUnIBERTI, Le fondement de l'effet des jugements étrangers, Leiden, Brill/Nijhoff, Académie de droit international de la Haye, 2019.

B. Hess/P. Ortolani (Eds.), Impediments of national procedural law to the free movement of judgments, München, C.H. Beck, Oxford, Hart, Baden-Baden, Nomos, 2019.

\section{Ley aplicable al proceso con elemento extranjero y asistencia judicial internacional}

N.M. Alexander/S. Chong, The Singapore Convention on Mediation: a commentary, Alphen aan den Rijn, Wolters Kluwer, 2019. 
I. BENÖHR, "Collective redress in the financial sector and the New EU Deal for Consumers", European Review of Private Law, 27, 6, 2019, pp. 1345-1366.

A.B. Biard/X.E. Kramer, "The EU Directive on Representative Actions for Consumers: a milestone or another missed opportunity", ZEuP, 27, 2, 2019, pp. 249-259.

R. CAPONI, "Ultime dall'Europa sull'azione di classe (con sguardo finale sugli Stati Uniti e il Dieselgate)", Foro it., CXLIV, 9, 2019, parte V, col. 332-338.

J. Chabrny, Grenzüberschreitende Sammelklagen, Hamburg, Verlag Dr. Kovač, 2019.

T. DomEJ, "Die geplante EU-Verbandsklagenrichtlinie: Sisyphos vor dem Gipfelsieg?", ZEuP, 27, 3, 2019, pp. 446-471.

M. Drehsen, "Zustellung gerichtlicher Schriftstücke im Rahmen der EuMahnVO”, IPRax, 39, 5, 2019, pp. 378-385.

F. Gascón Inchausti/M. StüRner, "Access to information and evidence in the ELI/Unidroit European rules on civil procedure: some fundamental aspects", Uniform Law Review, 24, 1, 2019, pp. 14-41.

W. HaKenberg/E.-M. KowoldiK, "New Deal for Consumers: europäische Kollektivklage und andere Wege moderner Durchsetzung von Verbraucherrechten", EWS, 30, 2, 2019, pp. 61-69.

R. JANSEN, "Explaining the methods for taking evidence abroad within the EU and some first observations on the proposal for the evidence regulation (recast)", NIPR, 37, 4, 2019, pp. 753-770.

F.J. JIMÉNEZ FORTEA (COORD.), La cooperación jurídica internacional civil y mercantil española más allá de la UE, Valencia, Tirant lo Blanch, 2019.

F. KLEIN, Die Verwertbarkeit gem. 28 USC § 1782(a) erlangter Beweismittel im deutschen Zivilprozess, Tübingen, Mohr Siebeck, 2019.

T.B. LÜHMANN, "Der Vorschlag einer europäischen Verbandsklage: ein weiteres Instrument des kollektiven Rechtsschutzes", $N J W, 72,9$, 2019, pp. 570-575.

T.B. LÜHMANN, "Risiken und Nebenwirkungen des IPR bei der kollektiven Durchsetzung von Kartellschadensersatzansprüchen: zur Bestimmung des anwendbaren Rechts bei Kartellschadensersatzansprüchen wegen Verletzung von europäischem und drittstaatlichem Kartellrecht”, $R I W, 65,1-2$, 2019, pp. 7-20.

A. Maffeo, Diritto dell 'Unione Europea e processo civile nazionale, Napoli, Editoriale Scientifica, 2019.

C. Peraro, "Legittimazione ad agire di un'associazione a tutela dei consumatori e diritto alla protezione dei dati personali a margine della sentenza Fashion ID", RDIPP, LV, 4, 2019, pp. 982-999.

C. Sсотт, "Consumer law, enforcement and the New Deal for Consumers", European Review of Private Law, 27, 6, 2019, pp. 1279-1296.

L. SERAFINELLI, "Ancora sulla tutela del consumatore, anche in forma collettiva”, La nuova giurisprudenza civile commentata, XXXV, 3, 2019, parte II, pp. 612-620.

V. SмIтн, "Redress through collective actions in Europe: ELI/Unidroit and European Commission proposals", Uniform Law Review, 24, 1, 2019, pp. 1-13.

C. WARIN, "Individual rights and collective interests in EU law: three approaches to a still volatile relationship", CMLR, 56, 2, 2019, pp. 463-487.

M. ZINNER/M. ReIFFENSTEIN, Hindernisse bei der kollektiven Verbraucherrechtsdurchsetzung: wie kann europäisches Recht gegensteuern?, Wien, Verlag Österreich, 2019.

\section{Derecho de los negocios internacionales}

S. BARIATTI, "Volontà delle parti e internazionalità del rapporto giuridico: alcuni sviluppi recenti nella giurisprudenza della Corte di giustizia sui regolamenti europei in materia di diritto internazionale privato", RDIPP, LV, 3, 2019, pp. 513-534.

V. DE BEAUFORT, Droit européen des affaires et politiques européennes, Bruxelles, Larcier, 2019.

J. ChuAh, Law of international trade: cross-border commercial transactions, $6^{\text {a }}$ ed., London, Sweet \& Maxwell, 2019.

C. Devaux, La fabrique du droit du commerce international: réguler les risques de capture, Bruxelles, Bruylant, 2019. 
D. EHLERS, "Grundrechtsbindung und Grundrechtsschutz von Unternehmen im deutschen und europäischen Recht”, DVBl., 134, 7, 2019, pp. 397-406.

M. HeIdemann, Transnational commercial law, London, Macmillan International Higher Education, Red Globe Press, 2019.

J. Honst, Transnationale Rechtserzeugung: Elemente einer normativen Theorie der Lex Financiaria, Tübingen, Mohr Siebeck, 2019.

P. Mankowski (Ed.), Commercial Law: article-by-article commentary, Baden-Baden, Nomos, München, Beck, Oxford, Chicago, Hart, 2019.

V. RéveILlère, "La conjonction des libertés de circulation et des droits fondamentaux", en Les libertés européennes de circulation au-delà de l'économie, Paris, Mare \& Martin, 2019, pp. 197-210.

\section{Competencia judicial internacional y negocios internacionales}

A.B. BiarD, "Justice en ligne, ou, Nouveau far www.est?: la difficile régulation des plateformes en ligne de règlement extrajudiciaire des litiges", Revue internationale de droit économique, 2, 2019, pp. 165-191

M. BRINKMANN, "Zum Zusammenhang zwischen Klage und Widerklage im europäischen Zivilprozessrecht (zu EuGH, 21.6.2018, Rs. C-1/17, Petronas Lubricants Italy SpA vs. Livio Guida)”, IPRax, 39, 6, 2019, pp. 501-506.

M. CAPOzzolo, Introduzione alla libera circolazione delle decisioni in materia civile e commerciale nello spazio giudiziario europeo: il regolamento (UE) n. 1215/2012 e gli altri regolamenti "dettoriali", Napoli, Editoriale scientifica, 2019.

K.S. DANELZIK, Die Gerichtsstandvereinbarung zwischen ZPO, EuGVVO und HGÜ, Baden-Baden, Nomos, 2019.

U. GRUŠIĆ, "Unjust enrichment and the Brussels I Regulation", ICLQ, 68, 4, 2019, pp. 837-868.

S. GuzZI, "Il regolamento sull'ordinanza europea di sequestro dei conti correnti bancari: tra mercato interno e prerogative della difesa", Il diritto dell'Unione europea, $\mathrm{n}^{\circ}$ 4, 2019, pp. 661-687.

B. HAFTEL, "Revirement et extension du champ de la 'matière contractuelle' dans les relations à trois personnes: CJUE, 7 mars 2018, no. C-274/16, C-447/16 et C-448/16, Flightright, CJUE, 4 oct. 2018, no. C-337/17, Feniks", Revue des contrats, 1, 2019, pp. 85-89.

W.J. HAU, "Rechtshängigkeitssperre durch obligatorische ADR-Verfahren im europäischen Zivilprozessrecht: Entscheidung des EuGH vom 20.12.2017: mit Anmerkung", ZEuP, 27, 2, 2019, pp. 384394.

C. KLEINER, “Compétence judiciaire: CJUE, 1re ch., 15 nov. 2018, aff. C-308/17, Hellenische Republik c/ Leo Kuhn”, JDI Clunet, 146, 3, 2019, pp. 854-867.

C. KOHLER, "Zur Rechtshängigkeit eines von der Zivilpartei vor dem Unterschungsrichter geltend gemachten Ersatzanspruchs (zu EuGH, 22.10.2015, Rs. C-523/14, Aannemingsbedrijf Aertssen NV u.a. vs. VSB Machineverhuur BV u.a., unten S. 147, Nr. 10)", IPRax, 39, 2, 2019, pp. 120-123.

S. Lee/M. PhuA, "Why Allianz v West Tankers still applies under the Brussels Regulation (Recast): an analysis of Nori Holdings v Bank Otkritie (2018) EWHC 1343 (Comm)", Journal of International Dispute Settlement, 10, 4, 2019, pp. 520-541.

V. LEgRAND, "Vers l'instrumentalisation des règles de litispendance européenne? (à propos de la décision de la CJUE, 16 janv. 2019, no. C-386/17, Liberato)", Petites affiches, 408, 53, 2019, pp. 7-11.

C. Lenz, Die Auswirkungen des référé-expertise auf deutsche Zivilgerichtsverfahren - die Brüssel-la-VO auf dem Prüfstand, Baden-Baden, Nomos, 2019.

Q.C. LOBACH, "Internationale Zuständigkeit der Gerichte am Erfüllungsort des Beförderungsvertrags für Ausgleichsansprüche des Fluggasts aus der Fluggastrechte-VO gegen einen vertragsfremden Teilstreckenbeförderer (zu EuGH, 7.3.2018, C-274/16, C-447/16 und C-448/16, flightright GmbH vs. Air Nostrum, Líneas Aéreas del Mediterráneo, SA u.a., unten S. 421, Nr. 39)", IPRax, 39, 5, 2019 , pp. 391-397.

M. LóPEZ De Gonzalo, "Quali (e quanti) fori per le controversie in materia di trasporto?", Diritto del commercio internazionale, 33.1, 2019, p. 161-170. 
M. LÓPEZ DE GoNZALO, "Spazio giudiziario europeo e forum non conveniens: una difficile convivenza", Diritto del commercio internazionale, 33.4, 2019, pp. 841-850.

F. MAILHÉ, "Un jeu d'asymétries: clauses d'éléction de for optionnelles et asymétriques dans la jurisprudence française", RIDC, 71, 2, 2019, pp. 431-446.

P. Mankowski, "Griechische Staatsanleihen und der griechische Schuldenschnitt vor dem EuGH: zugleich Besprechung EuGH v. 15.11.2018, Rs. C-308/17, ZIP 2018, 2290, Kuhn, (Folge Zwei)", ZIP, 40, 5, 2019, pp. 193-202.

M. Mantovani, "Notaries and their debt-collection writs under the Brussels Ia Regulation: a difficult characterisation", Journal of Private International Law, 15, 2, 2019, pp. 393-417.

P. Marcisz/A. Orzet-JaKuBowsKa, "The right to be unheard: recognition and enforcement of anti-suit injunctions issued by arbitrations in the EU", Journal of International Dispute Settlement, 10, 1, 2019, pp. 1-22.

A.R. Markus, "Rechtshängigkeit und Schlichtungsverfahren nach EuGH, C-467/16, Schlömp", GPR, 16, 2, 2019, pp. 60-66.

J. OsteR, “Ist Art. 5 Abs. 1, 1a EuGFVO grundrechtskonform?”, Zeitschrift für Zivilprozess international, 23, 2018 (2019), pp. 137-158.

L. Penasa, "Litispendenza e connessione extraeuropee nel Regolamento Bruxelles I-bis", Rivista di diritto processuale, LXXIV, 3, 2019, pp. 717-748.

S. PhuRTAG, Vorsorgliche Massnahmen im internationalen Zivilprozessrecht: unter besonderer Berücksichtigung des schweizerischen und englischen Rechts, Bern, Stämpfli Verlag, 2019.

A. PiekenBrock/D. Rodi, "Der Klägergerichtsstand des Unternehmers am Erfüllungsort bei Geldschulden: zugleich Anmerkung zu EuGH, Urt. v. 3.4.2019, C-266/18, Aqua Med sp. z o.o.”, GPR, 16, 5, 2019, pp. 233-240.

F. RIES, "Die Auslegung des Luganer Parallelübereinkommens nach der EuGVVO-Novelle: eine Untersuchung am Beispiel der Regelungen zu Gerichtsstandsvereinbarungen", RIW, 65, 1-2, 2019, pp. 32-36.

N. Ritz, Vorläufige Kontenpfändung in Europa, Berlin, Peter Lang, 2019.

D. Schnichels/K. LenZing/A. Stein, "Die Entwicklung des europäischen Zivilprozessrechts im Bereich der EuGVVO im Jahr 2018”, EuZW, 30, 21, 2019, pp. 885-894.

P. SiaPlaOURAS, "Gerichtsstandsvereinbarungen und Streitgenossenschaft im europäischen Zivilprozessrecht: zugleich Anmerkung zu EuGH, Urt. v. 28.6.2017, C-436/16, Leventis und Vafeias, ECLI:EU:C:2017:497”, GPR, 16, 1, 2019, pp. 13-19.

M. StÜRNER/F. PFÖRTNER, "Residual jurisdiction: back to the future?", GPR, 16, 5, 2019, pp. 222-228.

B. SUJECKI, "Rechtsprechung des Europäischen Gerichtshofs zur EuGVVO im Jahr 2018", EWS, 30, 4, 2019, pp. 196-206.

L. TichÝ (ED.), Standard of proof in Europe, Tübingen, Mohr Siebeck, 2019.

F. VISMARA, "La materia fiscale e doganale come limite materiale all'applicazione del Regolamento Bruxelles I-bis", RDIPP, LV, 1, 2019, pp. 114-126.

G. Vitellino, "Litispendenza e accordi confliggenti di scelta del foro nel caso BNP Paribas c. Trattamento rifiuti metropolitani”, RDIPP, LV, 4, 2019, pp. 999-1016.

\section{Sociedades de capital y otras personas jurídicas}

A.BRIGUglio, "Questioni didirittointernazionaleprivatoin materiadiresponsabilità direttadell'amministratore nei confroni del socio", Rivista di diritto societario, $\mathrm{n}^{\mathrm{o}}$ 1, 2019, parte III, pp. 223-248.

M. FALLON, "L'attractivité internationale comme moteur des projects de loi sur la BIBC et sur le futur Code des sociétés et associations au regard du droit international privé", Revue de droit commercial belge, 125, 1, 2019, pp. 7-51.

C. Gerner-Beuerle/F.M. Mucciarelli/E. Schuster/M. Siems (Eds.), The private international law of companies in Europe, München, Beck, Oxford, Chicago, Hart, Baden-Baden, Nomos, 2019.

J.C. GRÖNING, Gesellschafter- und Geschäftsleiterhaftung im internationalen Zivilverfahrensrecht: zur internationalen Zuständigkeit nach Brüssel Ia-VO und EuInsVO, Tübingen, Mohr Siebeck, 2019. 
S. Jung/P. Krebs/S. Stiegler (HrsG.), Gesellschaftsrecht in Europa: Handbuch, Baden-Baden, Nomos, 2019.

A. Mann, Britischer EU-Austritt und Scheinauslandsgesellschaften: Auswirkungen und Gestaltungsmöglichkeiten, Baden-Baden, Nomos, 2019.

S. ReUTER, "Grenzüberschreitende Gesellschaftervereinbarungen", RIW, 65, 1-2, 2019, pp. 21-32.

S. SAKKA, Der Konzern im Kompetenzrecht der EuGVVO: Unternehmensgruppe und internationale Zuständigkeit, Berlin, Peter Lang, 2019.

O. SснотеL, "International jurisdiction in cross-border corporate litigation", $N I P R, 37,2,2019$, pp. 350372.

P. SiaPlaOuRAS, "Gerichtsstandsvereinbarungen und Streitgenossenschaft im europäischen Zivilprozessrecht: zugleich Anmerkung zu EuGH, Urt. v. 28.6.2017, C-436/16, Leventis und Vafeias, ECLI:EU:C:2017:497”, GPR, 16, 1, 2019, pp. 13-19.

G. Vitellino, "Note a margine di una pronuncia del tribunale di Torino in materia societaria", RDIPP, LV, 4, 2019, pp. 1016-1028.

\section{Contratos internacionales (I)}

S. AMPATZI, Das AGB-Recht in der nationalen und internationalen Schiedsgerichtsbarkeit im unternehmerischen Geschäftsverkehr: eine Untersuchung im deutschen und griechischen Recht, BadenBaden, Nomos, 2019.

M. Bнатт, Islamic law and international commercial arbitration: The application of shari 'a in contemporary international commercial arbitration, London, New York, Routledge, 2019.

S. BIEHL, Eingriffsnormen und Schiedsvereinbarungen: eine Untersuchung der Auswirkungen von Eingriffsnormen auf die Anerkennung einer internationalen Schiedsvereinbarung in der Einredesituation, Berlin, Peter Lang, 2019.

J. BöHLE, "Die Abwahl zwingenden Rechts vor staatlichen Gerichten in Inlandsfällen", ZEuP, 27, 1, 2019, pp. 72-98.

R. CaValieri/V. Salvatore, An introduction to international contract law, $2^{\mathrm{a}}$ ed., Torino, G. Giappichelli editore, 2019.

J.M. Espinar Vicente/J.I. Paredes Pérez, El régimen jurídico de las obligaciones en Derecho internacional privado español y de la Unión Europea, Madrid, Dykinson, 2019.

P. FABIG, "Der Vertragshändlerausgleich in internationalen Verträgen”, $I H R, 19,1,2019$, pp. 1-11.

S. FEILER, Kollisionsrecht der Vertragsübernahme, Baden-Baden, Nomos, 2019.

S. GössLING, Europäisches Kollisionsrecht und internationale Schiedsgerichtsbarkeit: die Bedeutung der Rom I-Verordnung bei der Bestimmung des anwendbaren materiellen Rechts durch internationale Handelsschiedsgerichte mit Sitz in der EU, Tübingen, Mohr Siebeck, 2019.

C. HARMs, Neuauflage der Datumtheorie im Internationalen Privatrecht, Tübingen, Mohr Siebeck, 2019.

A.N. KaRADAYI Yalim, Interpretation and gap filling in international commercial contracts, Cambridge, Antwerp, Chicago, Intersentia, 2019.

W.J. KASSIR, Autonomie de la volonté et contrat sans loi en droit international privé des contrats: consécration mondiale, risques d'exacerbation et nécessité de préserver l'autorité de la loi dans les contrats internationaux, Beyrouth, Point Delta, Issy-les-Moulineaux, LGDJ, 2019.

P. Mankowski, "Politik und missliebige drittstaatliche Eingriffsnormen: der Fall Kuwait Airways: zugleich Besprechung zu OLG Frankfurt, RIW 2019, 231", RIW, 65, 4, 2019, pp. 180-184.

G. Passarelli, Contract law in contemporary international commerce: considerations on the complex relationship between legal process and market process in the new era of globalisation, BadenBaden, Nomos, 2019.

R. Pereira Dias, "Auslandsbezug des Sachverhalts nach Art. 3 Abs. 3 EVÜ/Rom I-VO und rebus sic stantibus-Klausel des portugiesischen Zivilrechts als nicht parteidisponible Bestimmung: Entscheidung des englischen Court of Appeal vom 13.Dezember 2016: mit Anmerkung", ZEuP, 27, 3, 2019, pp. 603-619. 
E.A. Rossi, La sharing economy nel diritto internazionale privato europeo, Torino, G. Giappichelli editore, 2019.

G. SchefFER DA SilveIRA, Les modes de règlement des différends dans les contrats internationaux de construction, Bruxelles, Bruylant, 2019.

T. SzaBAdos, Economic sanctions in EU private international law, Oxford, Hart, 2019.

M. THON, "Forumfremde Eingriffsnormen und Außenpolitik im IPR: die Israel-Boykottgesetzgebung arabischer Staaten und ihre Berücksichtigung vor deutschen Gerichten (zu OLG Frankfurt a.M., 25.9.2018, 16 U 209/17, unten S. 321, Nr. 30)", IPRax, 39, 4, 2019, pp. 301-306.

P. WaUtelet/A. Ernoux, "Le droit international privé et les relations contractuelles qui se nouent par le biais des plateformes", en Enjeux et défis juridiques de l'économie de plateforme, 2019, pp. 34-84.

\section{Contratos internacionales (II). Contratos en particular}

M.-É. ANCEL, "La loi applicable à l'opposabilité aux tiers des cessions de créances: propos introductifs", DA: revue internationale du droit des affaires, 1, nº 129, 2019, pp. 14-18.

L. De BAERE/F. BleEs, Insurance aspects of cross-border road traffic accidents, The Hague, the Netherlands, Eleven International Publishing, 2019.

C. BAmford, Principles of international financial law, $3^{\mathrm{a}}$ ed., Oxford, Oxford University Press, 2019.

D. Brauner, Die Anwendungsbereiche von CISG und PR CESL im Vergleich, Berlin, Lang, 2019.

G. CAVALIER, "Règle fiscale française, droit international privé (communautaire) et droit (non fiscal) étranger: quelle méthode d'articulation?", Revue internationale des services financiers, $\mathrm{n}^{\circ} 1,2019$, pp. 82-91.

D. EINSELE, "Die Drittwirkung von Forderungsübertragungen im Kollisionsrecht: ein kritischer Zwischenruf zum Verordnungsvorschlag der Kommission", IPRax, 39, 6, 2019, pp. 477-483.

S. FeILER, Kollisionsrecht der Vertragsübernahme, Baden-Baden, Nomos, 2019.

M. Fras, "The group insurance contract in private international law", NILR, 66, 3, 2019, pp. 507-535.

D. GeSMAnN-NuIssL, Internationales Franchise-Recht, Frankfurt am Main, Fachmedien Recht und Wirtschaft, 2019.

F. HeINDLER, "The law applicable to third-party effects of transactions in intermediated securities", Uniform Law Review, 24, 4, 2019, pp. 685-723.

C.A. HeInZE/C.J. W ARMUTH, "The law applicable to third-party effects of assignment and the conflict rules for insolvency according to EU law", Uniform Law Review, 24, 4, 2019, pp. 664-684.

L. HÜBNER, "Die Drittwirkungen der Abtretung im IPR: ein weiterer Schritt zur Fortentwicklung des europäischen Kollisionsrechts", ZEuP, 27, 1, 2019, pp. 41-71.

E.-M. KIENINGER, "European rules on the law applicable to third-party effects of assignments: a neverending story?", Uniform Law Review, 24, 4, 2019, pp. 633-648.

E.-M. KIENINGER, "Das auf die Drittwirkungen der Abtretung anwendbare Recht: der EuGH spielt den Ball zurück nach Deutschland", $N J W, 72,46,2019$, pp. 3353-3356.

M. LAAzouzI, "L'éviction du for de l'action directe de la victime au profit de l'arbitrage international prévu par le contrat d'assurance?", Revue des contrats, 4, 2019, pp. 69-73.

G. MinNe, "Le gage et le transfert de propriété à titre de garantie dans la proposition de règlement européen sur la loi applicable à l'opposabilité des cessions de créances", DA: Revue internationale du droit des affaires, 1, no 129, 2019, pp. 35-47.

D.-M. PhiLIPPE, "La proposition de règlement du Parlement européen et du Conseil sur la loi applicable à l'opposabilité des cessions de créances: aspects liés à la cession de créance elle-même", DA: Revue internationale du droit des affaires, 1, no 129, 2019, pp. 19-26.

J.-P. Spang, "La proposition de règlement du Parlement européen et du Conseil sur la loi applicable à l'opposabilité des cessions de créances: aspects liés à la titrisation", DA: Revue internationale du droit des affaires, $1, \mathrm{n}^{\mathrm{o}} 129,2019$, pp. 27-34.

S. WALTER, "Die Abdingbarkeit zwingenden Handelsvertreterrechts und die Handelsvertreter-Richtlinie: das Agro-Missverständnis", RIW, 65, 9, 2019, pp. 570-576. 
C. Wendland, "Abtretungen und Verbraucherschutz unter der Rom I-Verordnung: zessionsbedingte Neuanknüpfung von Verbrauchervertragsanforderungen?”, ZVglRWiss, 118, 4, 2019, pp. 422-448.

P.R. Wood, Conflict of laws and international finance, $2^{\mathrm{a}}$ ed., London, Sweet \& Maxwell, 2019.

\section{Contratos internacionales de consumo}

I. BENÖHR, "Collective redress in the financial sector and the New EU Deal for Consumers", European Review of Private Law, 27, 6, 2019, pp. 1345-1366.

A.B. BIARD, "Towards high-quality consumer ADR: the Belgian experience", en Privatizing dispute resolution: trends and limits, Baden-Baden, Nomos, 2019, pp. 79-103.

A.B. Biard/X.E. Kramer, "The EU Directive on Representative Actions for Consumers: a milestone or another missed opportunity", ZEuP, 27, 2, 2019, pp. 249-259.

F. BRAUN, "Das Verbraucherstreitbeilegungsgesetz im Konzert der Verbraucherrechtsdurchsetzung", Verbraucher und Recht, 34, 4, 2019, pp. 130-135.

G.-P. CALLIESS, Grenzüberschreitende Verbraucherverträge: Rechtssicherheit und Gerechtigkeit auf dem elektronischen Weltmarktplatz, Tübingen, Mohr Siebeck, 2019.

J. Chabrny, Grenzüberschreitende Sammelklagen, Hamburg, Verlag Dr. Kovač, 2019.

J.M.L. Duin/C. van LeONE, "The real (new) deal: levelling the odds for consumer-litigants: on the need for modernization. Part II", European Review of Private Law, 27, 6, 2019, pp. 1227-1249.

W. HaKenBerg/E.-M. Kowollik, "New Deal for Consumers: europäische Kollektivklage und andere Wege moderner Durchsetzung von Verbraucherrechten", EWS, 30, 2, 2019, pp. 61-69.

J. HaSlaCh, "International jurisdiction in consumer contract cases under the Brussels I Regulation: Schrems: case C-498/16, Maximilian Schrems v. Facebook Ireland Limited, judgment of the Court (Third Chamber) of 25 January 2018, EU:C:2018:37”, CMLR, 56, 2, 2019, pp. 559-579.

B. Hess/S. Law (Eds.), Implementing EU consumer rights by national procedural law, München, C.H. Beck, Oxford, Hart, Baden-Baden, Nomos, 2019.

F. HIDDING, Zugang zum Recht für Verbraucher: ein Vergleich der alternativen Streitbeilegung in Verbrauchersachen mit der gerichtlichen Streitbeilegung, Berlin, Duncker \& Humblot, 2019.

M. Ho-DAC, "Précisions sur l'office du juge (d'origine) en matière d'exécution internationale des décisions dans les litiges européens de consommation: CJUE, 4 septembre 2019, Salvoni, C-347/18, ECLI:EU:C:2019:661", Revue des affaires européennes, 24, 3, 2019, pp. 653-662.

A. KAMPHORSt, "The right to a fair trial in online consumer dispute resolution", Journal of European Consumer and Market Law, 8, 5, 2019, pp. 175-183.

M.B.M. Loos, "Towards civil justice in the EU: the European Commission's New Deal for Consumers: an introduction to this issue", European Review of Private Law, 27, 6, 2019, pp. 1219-1225.

C. Meller-Hannich, "Sammelklagen am europäischen Verbrauchergerichtsstand?: Urteil des Gerichtshofs (Dritte Kammer) vom 25. Januar 2018: mit Anmerkung”, ZEuP, 27, 1, 2019, pp. 205-212.

E.A. OnȚANU, "Court and out-of-court procedures: in search of a comprehensive framework for consumers' access to justice in cross-border litigation", en Privatizing dispute resolution: trends and limits, Baden-Baden, Nomos, 2019, pp. 49-78.

C. PAVILlon, "Private enforcement as a deterrence tool: a blind spot in the Omnibus-Directive", European Review of Private Law, 27, 6, 2019, pp. 1297-1328.

C. Peraro, "Legittimazione ad agire di un'associazione a tutela dei consumatori e diritto alla protezione dei dati personali a margine della sentenza Fashion ID", RDIPP, LV, 4, 2019, pp. 982-999.

G. Risso, "Product liability and protection of EU consumers: is it time for a serious reassessment?", Journal of Private International Law, 15, 1, 2019, pp. 210-233.

C. Sсотт, "Consumer law, enforcement and the New Deal for Consumers", European Review of Private Law, 27, 6, 2019, pp. 1279-1296.

L. SERAFINELLI, "Ancora sulla tutela del consumatore, anche in forma collettiva", La nuova giurisprudenza civile commentata, XXXV, 3, 2019, parte II, pp. 612-620. 
B.A. WARWAS, "Access to privatized consumer justice: arbitration, ADR, and the future of value-oriented justice in the EU", en Privatizing dispute resolution: trends and limits, Baden-Baden, Nomos, 2019, pp. 325-347.

C. Wendland, "Abtretungen und Verbraucherschutz unter der Rom I-Verordnung: zessionsbedingte Neuanknüpfung von Verbrauchervertragsanforderungen?", ZVglRWiss, 118, 4, 2019, pp. 422-448.

J.J. Witte/M.S. WetZIG, "Die Musterfeststellungsklage, Placebo oder Allheilmittel für den deutschen Verbraucherschutz?: ein Kommentar zur Einführung der Musterfeststellungsklage aus rechtsvergleichender Sicht", $W M, 73,2,2019$, pp. 52-59.

M. ZINNER/M. ReIFFENSTEIN, Hindernisse bei der kollektiven Verbraucherrechtsdurchsetzung: wie kann europäisches Recht gegensteuern?, Wien, Verlag Österreich, 2019.

\section{Contrato internacional de trabajo}

U. BECKER, "Grundrechte der Arbeit in Europa: zu Funktionen, Verschränkungen und Konfliktlinien vernetzter Grundrechtsordnungen", EuR, 54, 5, 2019, pp. 469-501.

U. GRuŠIĆ, "L'effet du Brexit sur le droit international privé du travail", RCDIP, 2019, pp. 367-384.

B. HAFTEL, "Contrat de travail, salarié étranger en situation irrégulière et lois de police: le Conseil d'État se convertit, un peu trop, aux méthodes du droit international privé", Revue des contrats, 4, 2019, pp. 65-68.

A. JUNKER, Internationales Arbeitsrecht im Konzern, Tübingen, Mohr Siebeck, 2019.

O.L. KNÖFEL, "Flugpersonal im europäischen Arbeitsprozessrecht: zugleich Anmerkung zu EuGH, Urt. v. 14.9.2017, verb. C-168/16 u. C-169/16 (Nogueira u.a. vs. Crewlink Ireland Ltd. u. Moreno Osacar vs. Ryanair Designated Activity Company, vormals Ryanair Ltd.)", GPR, 16, 1, 2019, pp. 43-48.

A. NUYTS, "L'affaire Ryanair: le for compétent pour les litiges avec le personnel de cabine: note", Revue de droit commercial belge, 125, 1, 2019, pp. 84-90.

D. OUdKerk/A. Rogers \& Contributors, International employment law disputes, London, Sweet \& Maxwell, 2019.

M.E. RISAK, "Grenzüberschreitendes Arbeiten im virtuellen Raum: ein Fall für das europäische Arbeitsrecht?", Das Recht der Arbeit, 69, 2, 2019, n 381, pp. 117-121.

F. ThомAs, Les relations de travail offshore: contribution à l'étude du pluralisme juridique, Aix-enProvence, Presses universitaires d'Aix-Marseille, 2019.

G.W. VAN DER VOET, "International maritime employment law: about the special legal status of the most globalised of international workers: the seafarer", NIPR, 37, 1, 2019, pp. 4-20.

\section{Derechos reales}

Z. Crespi Reghizzi, "Profili di diritto internazionale privato del commercio dei beni culturali", Diritto del commercio internazionale, 33.2, 2019, pp. 361-384.

M. Frigo, "La transition en droit interne italien de la Directive 2014/60 relative à la restitution de biens culturels ayant quitté illicitement le territoire d'un État membre", en Un patrimoine vivant, entre nature et culture: liber amicorum en l'honneur de Jérôme Fromageau, Paris, Mare \& Martin, 2019, pp. 327-339.

P. Hartmann, Sicherheitsübertragungen und besitzlose Pfandrechte im europäischen Insolvenzrecht: unter besonderer Berücksichtigung des deutschen, englischen und französischen Rechts, Köln, Heymanns, 2019.

G. Minne, "Le gage et le transfert de propriété à titre de garantie dans la proposition de règlement européen sur la loi applicable à l'opposabilité des cessions de créances", DA: Revue internationale du droit des affaires, 1, nº 129, 2019, pp. 35-47. 


\section{Propiedad intelectual e industrial}

R.M. Ballardini/P. Kuoppamäki/O. PitKänen (Eds.), Regulating industrial internet through IPR, data protection and competition law, Alphen aan den Rijn, Kluwer Law International, 2019.

Y. BASIRE, "La propriété industrielle devant les juridictions de l'Union européenne (novembre 2017-novembre 2018)", Europe, 29, 3, 2019, pp. 13-17.

T. DreiER, "Grundrechte und die Schranken des Urheberrechts: Anmerkung zu EuGH 'Funke Medien NRW' und 'Spiegel Online'”, GRUR Int., 68, 10, 2019, pp. 1003-1008.

A. ECKERT, Die Durchsetzung von Benutzungsverboten im Unionsdesign- und Unionsmarkenrecht: Zwangsgeldvollstreckung im Europäischen Rechtsraum, Jena, JWV Jenaer Wissenschaftliche Verlagsgesellschaft, 2019.

F. EICHEL, "Der prozessuale Handlungsort bei internationalen Markenrechtsverletzungen im Internet (zu BGH, 9.11.2017, I ZR 164/16, unten S. 48, Nr. 1, und öst. OGH, 20.12.2016, 4 Ob 45/16w, unten S. 53, Nr. 2)", IPRax, 39, 1, 2019, pp. 16-23.

M. Goldhammer, "Realitäten europäischer Kommunikationsgrundrechte und der lange Arm des Urhebers: Anmerkung zu EuGH, Urteil vom 29.7.2019, C-516/17, Spiegel Online/Beck (ZUM 2019, 759)", ZUM, 63, 10, 2019, pp. 727-731.

J. GRIFFITHS, "European Union copyright law and the Charter of Fundamental Rights: Advocate General Szpunar's opinions in (C-469/17) Funke Medien, (C-476/17) Pelham GmbH and (C-516/17), Spiegel Online", ERA-Forum, 20, 1, 2019, pp. 35-50.

P. Homar, "Umsetzung und Anwendung der Urheberrechtsausnahmen: zugleich eine Anmerkung zu EuGH 29.07.2019, C-516/17, 'Spiegel Online'”, Medien und Recht, 16, 3-4, 2019, pp. 79-89.

P. Homar, "Enge Handlungsspielräume für das Sampling: Anmerkung zu EuGH, Urteil vom 29.7.2019, C-476/17, Pelham u.a./Hütter u.a. (ZUM 2019, 738)”, ZUM, 63, 10, 2019, pp. 731-737.

S. HorN, "Grenzüberschreitende Musterfeststellungsklagen", ZVglRWiss, 118, 3, 2019, pp. 314-340.

M. Leistner, "Das Urteil des EuGH in Sachen 'Funke Medien NRW/Deutschland': gute Nachrichten über ein urheberrechtliches Tagesereignis: Anmerkung zu EuGH, Urteil vom 29.7.2019, C-469/17, Funke Medien NRW/Deutschland (ZUM 2019, 751)”, ZUM, 63, 10, 2019, pp. 720-726.

D. Moura Vicente, A tutela internacional da propriedade intelectual, $2^{\mathrm{a}}$ ed., Coimbra, Almedina, 2019.

K. RANTASAARI, "The European multiple patent defendants rule as a legal constraint for start-ups and growth companies", Journal of Intellectual Property Law \& Practice, 14, 12, 2019, pp. 949-957.

J. SchULTE, "Gemeinsam verzockt: Zuständigkeit und Anknüpfung bei Verletzung eines Gemeischaftsgeschmacksmusters durch mehrere Schädiger (zu EuGH, 27.9.2017, Rs. C-24, 25/16, Nintendo, unten S. 233, Nr. 18)", IPRax, 39, 3, 2019, pp. 202-208.

C. SGANGA, "The fundamental rights saga in EU copyright law: time for the boundary-setting season?", Medien und Recht, 16, 2, 2019, pp. 56-63.

C. SGANGA, "A decade of fair balance doctrine, and how to fix it: copyright versus fundamental rights before the CJEU from Promusicae to Funke Medien, Pelham and Spiegel online”, EIPR, 41, 11, 2019, pp. 683-696.

M. STIEPER, "Der Trans Europa Express ist aus Luxemburg zurück: auf dem Weg zu einer Vollharmonisierung der urheberrechtlichen Schranken: zugleich Anmerkung zu EuGH, Urteile vom 29.7.2019, C-469/17, C-476/17, C-516/17 (ZUM 2019, 751, Funke Medien NRW/Deutschland, ZUM 2019, 738, Pelham u.a./Hütter u.a. und EuGH 2019, 759, Spiegel Online/Beck)”, ZUM, 63, 10, 2019, pp. 713-720.

T.E. Synodinou (ED.), Pluralism or universalism in international copyright law, Alphen aan den Rijn, Wolters Kluwer, 2019.

L. TochtermanN, "Joint liability in Germany for patent infringement committed abroad", Journal of intellectual property law \& practice, 14, 6, 2019, pp. 494-503.

É. Treproz, "Marques de l'Union et forum shopping", Revue des affaires européennes, 24, 3, 2019, 3, pp. 541-551.

J.J. Witte/M.S. WetZIG, "Die Musterfeststellungsklage, Placebo oder Allheilmittel für den deutschen Verbraucherschutz?: ein Kommentar zur Einführung der Musterfeststellungsklage aus rechtsvergleichender Sicht", $W M, 73,2,2019$, pp. 52-59. 
L. WolLENSChläGER, Die kollisionsrechtliche Anknüpfung des Patentvindikationsanspruches, Köln, Heymann, 2019.

\section{Obligaciones extracontractuales}

R. Amaro, "Le contentieux de la réparation des pratiques anti-concurrentielles (sept. 2018-juin 2019)", Concurrences, $\mathrm{n}^{\mathrm{o}} 3,2019$, pp. 230-246

E.-M. BAJONs, "OGH (contra EuGH): (kein) Amtshaftungsanspruch bei Verletzung von Aufsichtspflichten durch einen beamteten Lehrer", ZfRV, 60, 5, 2019, pp. 196-199.

P. BIROLLEAU, "La difficile conciliation avec le droit européen de la rupture brutale des relations d'affaires", Revue Lamy de la concurrence, $n^{\circ} 80,2019$, pp. 22-26.

D. BuREAU, "À propos du règlement Rome II: de la règle de conflit aux lois de police en passant par la transposition des directives (CJUE 31 janv. 2019, aff. C-149/18, D. 2019. 257, ibid. 1016, obs. S. Clavel et F. Jault-Seseke)", RCDIP, 2019, pp. 557-571.

R. CAPONI, "Ultime dall'Europa sull'azione di classe (con sguardo finale sugli Stati Uniti e il Dieselgate)", Foro it., CXLIV, 9, 2019, parte V, col. 332-338.

J. Chabrny, Grenzüberschreitende Sammelklagen, Hamburg, Verlag Dr. Kovač, 2019.

A. ENGEL, Internationales Kapitalmarktdeliktsrecht: eine Untersuchung zum anwendbaren Recht der Prospekthaftung und der Haftung für fehlerhafte Sekundärmarktinformation (insbesondere Adhoc-Publizität) in den USA und der EU, Tübingen, Mohr Siebeck, 2019.

M. FrançoIse, "Retour sur la qualification de loi de police d'une loi nationale de transposition et précisions quant au champ d'application matériel du règlement Rome II au regard des directives européennes: CJUE, 31 janv. 2019, no. C-149/18, Agostinho da Silva Martins c/ Dekra Claims Services Portugal SA", Petites affiches, 408, 85, 2019, pp. 15-18.

T. GÖRGEN, Unternehmerische Haftung in transnationalen Menschenrechtsfällen: eine Untersuchung der zivilrechtlichen Haftung unter besonderer Berücksichtigung der UN-Leitprinzipien für Wirtschaft und Menschenrechte, Baden-Baden, Nomos, 2019.

U. GRUŠIĆ, "Unjust enrichment and the Brussels I Regulation", ICLQ, 68, 4, 2019, pp. 837-868.

M. HABERSACK/M. EHRL, "Verantwortlichkeit inländischer Unternehmen für Menschenrechtsverletzungen durch ausländische Zulieferer: de lege lata und de lege ferenda", $A c P, 219,2$, 2019, pp. 155-210.

R. HACKBARTH, "Kehrtwende beim internationalen Tatortgerichtsstand?: Anmerkungen zur 'AMS Neve'Entscheidung", GRUR Int., 68, 12, 2019, pp. 1269-1273.

F. HeRRMANN/M. JAKOB, "Arbitration cartel damage claims in the EU: still possible after the CDC judgment?: an analysis on the arbitrability of cartel damage claims and the interpretation of the scope of arbitration agreements", Global Competition Litigation Review, 12, 3, 2019, pp. 118-129.

B. Hess, "Protecting privacy by cross-border injunction", RDIPP, LV, 2, 2019, pp. 284-301.

L. IDOT, "Le contentieux international des actions en répartition pour violation du droit de la concurrence: l'arrêt CDC revisité (CJUE, 2e ch., 5 juill. 2018, aff. C-27/17, AB 'fly-LAL-Lithuanian Airlines' [...])", RCDIP, 2019, pp. 786-816.

C. KLEINER, "For délictuel, localisation du préjudice financier (dévalorisation des titres financiers): CJUE, 1er ch., 12 sept. 2018, aff. C-304/17, Helga Löber c/ Barclays Bank plc., JurisData no. 2018017863", JDI Clunet, 146, 2, 2019, pp. 503-516.

T. KLICKA, "Die Anwendung des Deliktsgerichtsstands nach Art $7 \mathrm{Nr} 2$ EuGVVO auf reine Vermögensschäden eines Fahrzeugkäufers: eine Replik auf Oberhammer, JB1 2018, 750”, Juristische Blätter, 141, 6, 2019, pp. 337-346.

A. LANG, Internationale und interstaatliche Zuständigkeit US-amerikanischer Gerichte für Produkthaftungsklagen: personal jurisdiction in stream-of-commerce cases, Hamburg, Verlag Dr. Kovač, 2019.

M. LehmanN/F. Eichel, "Globaler Klimawandel und internationales Privatrecht: Zuständigkeit und anzuwendendes Recht für transnationale Klagen wegen klimawandelbedingter Individualschäden", RabelsZ, 83, 1, 2019, pp. 77-110. 
M. LENDERMANN, Strafschadensersatz im internationalen Rechtsverkehr: die Behandlung ausländischer punitive damages im deutsch-französischen Rechtsvergleich, Tübingen, Mohr Siebeck, 2019.

T.B. LÜHMANN, "Risiken und Nebenwirkungen des IPR bei der kollektiven Durchsetzung von Kartellschadensersatzansprüchen: zur Bestimmung des anwendbaren Rechts bei Kartellschadensersatzansprüchen wegen Verletzung von europäischem und drittstaatlichem Kartellrecht”, $R I W, 65,1-2$, 2019, pp. 7-20.

U. Magnus/P. Mankowski (Eds.), Rome II Regulation: Commentary. European commentaries on private international law, Köln, O. Schmidt, Sellier European Law Publishers, 2019.

P. MANKowski, "Reichweite von Gerichtsstandsvereinbarungen: kartellrechtliche Schadensersatzklage: EuGH, 24.10.2018, C-595/17 Apple Sales International, Apple Inc., Apple retail France EURL vs. MJA als Liquidator von eBizcuss.com: mit Anmerkung", JZ, 74, 2, 2019, pp. 141-144.

N. MichaIL, "Entre le RGPD et le règlement Bruxelles Ibis: quel juge pour protéger nos données personnelles?", Droit de la consommation, $\mathrm{n}^{\circ}$ 122-123, 2019, pp. 175-200.

H. MuiR-WatT, "Le retour du forum actoris en matière financière se précise (CJUE, 1re ch., 12 sept. 2018, aff. C-304/17, D. 2018.1761)", RCDIP, 2019, pp. 135-146.

C.S.A. Окоц//E. Roberts, "The operation of Article 4 of Rome II Regulation in English and Irish courts", Journal of Private International Law, 15, 3, 2019, pp. 605-625.

J. Oster, Kommunikationsdeliktsrecht: eine transnationale Untersuchung am Beispiel des Ehrschutzes, Tübingen, Mohr Siebeck, 2019.

L. Pailler, "Conflit de lois: CJUE, 6e ch., 31 janv. 2019, aff. C-149/18, Agostinho da Silva Martins c/ Dekra Claims Services Portugal SA", JDI Clunet, 146, 3, 2019, pp. 878-894.

L. RADEMACHER, "Favor laesi und renvoi: Verweisungsart bei Distanzdelikten (zu OLG Hamm, 3.5.2017, 3 U 30/17, unten S. 165, Nr. 17)", IPRax, 39, 2, 2019, pp. 140-147.

G. Risso, "Product liability and protection of EU consumers: is it time for a serious reassessment?", Journal of Private International Law, 15, 1, 2019, pp. 210-233.

R. SACK, Internationales Lauterkeitsrecht, Köln, Carl Heymanns Verlag, 2019.

I. SCHERER, "Prozessuale Fragestellungen beim 'Folgenbeseitigungsanspruch' im Lauterkeitsrecht", Verbraucher und Recht, 34, 7, 2019, pp. 243-251.

B.J. SCRABACK, "Marktortanknüpfung bei Kartellen?: Anmerkung zu EuGH, Urt. v. 5.7.2018, C-27/17, AB flyLAL, Lithuanian Airlines vs. Starptautiskā lidosta, Rīga VAS, Air Baltic Corporation AS, ECLI:EU:C:2018:533”, GPR, 16, 2, 2019, pp. 69-73.

P. SiaPlaOURAS, "Gerichtsstandsvereinbarungen und Streitgenossenschaft im europäischen Zivilprozessrecht: zugleich Anmerkung zu EuGH, Urt. v. 28.6.2017, C-436/16, Leventis und Vafeias, ECLI:EU:C:2017:497”, GPR, 16, 1, 2019, pp. 13-19.

A. Staudinger, "10 Jahre Rom II-VO und Haager Straßenverkehrsübereinkommen: Zeit für eine Bestandsanalyse im Lichte des Brexit und der angedachten EU-Erweiterung", Deutsches Autorecht, 89, 12, 2019, pp. 669-675.

T. ThIEDE/S. LoRsCHEIDER, "Die internationale Zuständigkeit für Ansprüche von Anlegern aus Prospekthaftung: zugleich Besprechung von EuGH, Urt. v. 12.9.2018, Löber/Barclays (C-304/17)", EuZW, 30, 7, 2019, pp. 274-280.

C. Thole, "Die Abgrenzung zwischen EuInsVO und EuGVVO bei Haftungsklagen gegen Dritte wegen eines Gläubigergesamtschadens (zu EuGH, 6.2.2019, Rs. C-535/17, NK, Konkursverwalter der PI Gerechtsdeurwaarderskantoor BV und von PI vs. BNP Paribas Fortis NV)", IPRax, 39, 6, 2019, pp. 483-488.

C.E. Tuo, "The consequences of Brexit for recognition and enforcement of judgments in civil and commercial matters: some remarks", RDIPP, LV, 2, 2019, pp. 302-308.

W. VAN DER VELDE, "Beachcombing for liability limits?: wreck and cargo removal claims in private international law", NIPR, 37, 1, 2019, pp. 21-33.

I. Welser/A.K. Wolf-Posch, "Private enforcement of cartel infringements before arbitral tribunals", Austrian Yearbook on International Arbitration, 2019, pp. 39-65.

C. WendelsteIn, “"Menschenrechtliche' Verhaltenspflichten im System des internationalen Privatrechts”, RabelsZ, 83, 1, 2019, pp. 111-153. 
P. Westerhoven, Gerichtsstandsklauseln in der privaten Durchsetzung des EU-Kartellrechts, BadenBaden, Nomos, 2019.

F. ZufaLl, "Shifting role of the 'place': from locus delicti to online ubiquity in EU, Japanese and U.S. conflict of tort laws", RabelsZ, 83, 4, 2019, pp. 760-796.

\section{Insolvencia internacional}

S. Balthasar, "Unternehmenssanierungen nach dem Schuldverschreibungsgesetz und der Restrukturierungsrichtlinie: eine kritische Analyse", $Z H R, 183,6,2019$, pp. 662-694.

L. BENEDETTI, "Information flows in the insolvency of enterprise groups", European Business Law Review, 30, 3, 2019, pp. 417-438.

D. BRAMKAMP, Die Attraktivgerichtsstände des europäischen Insolvenzrechts: eine Untersuchung der Regelungen des Art. 6 EulnsVO, Köln, Carl Heymanns Verlag, 2019.

M. BRINKMANN (ED.), European insolvency regulation: article-by-article commentary, München, Beck, Baden-Baden, Nomos, Oxford, Chicago, Hart, 2019.

D. BuREAU, "De la compétence exclusive en matière d'action révocatoire", RCDIP, 2019, pp. 476-486.

B. Dostal, "Zur Frage der internationalen Zuständigkeit und des anwendbaren Rechts für Gläubigeranfechtungsklagen: zugleich Anmerkung zur Entscheidung des EuGH vom 4.10.2018", IHR, 19, 3, 2019, pp. 89-101.

H. EIDENMÜLLER, "The rise and fall of regulatory competition in corporate insolvency law in the European Union", EBOR, 20, 3, 2019, pp. 547-566.

F. FRIND, "EU-Restrukturierungsrichtlinie: der insolvenzrechtliche Überschuldungstatbestand als notwendiges 'Frühwarnsystem' bei der nationalen Umsetzung”, BB, 74, 41, 2019, pp. 2381-2387.

D.F. Fritz/L. Scholtis, "Rechtsprechungsübersicht im internationalen Insolvenzrecht 2019/2019: Entscheidungen des EuGH und deutscher Gerichtsbarkeit", IWRZ, 4, 4, 2019, pp. 147-152.

F.J. Garcimartín AlfÉrez, "The EU regime on securitisation: coordination between the regulatory framework and the conflict of law rules", ZEuP, 27, 1, 2019, pp. 13-40.

E. GöRETZLeHner, Maritime Cross-Border Insolvency: An Analysis for Germany, England \& Wales and the USA, Cham, Springer, 2019.

F. GRELL/U. KLOCKENBRINK, "Auswirkungen der Richtlinie über präventive Restrukturierungsrahmen auf Fremdkapitalgeber: ausgewählte Themen von Cram Down bis Lender Liability”, $D B, 72,26,2019$, pp. 1489-1494.

J.C. GRÖNING, Gesellschafter- und Geschäftsleiterhaftung im internationalen Zivilverfahrensrecht: zur internationalen Zuständigkeit nach Brüssel Ia-VO und EuInsVO, Tübingen, Mohr Siebeck, 2019.

M. HAENTJENS/B. Wessels (EDS.), Research handbook on cross-border bank resolution, Cheltenham, UK, Northampton, MA, USA, Edward Elgar Publishing, 2019.

P. Hartmann, Sicherheitsübertragungen und besitzlose Pfandrechte im europäischen Insolvenzrecht: unter besonderer Berücksichtigung des deutschen, englischen und französischen Rechts, Köln, Heymanns, 2019.

B. Hess (HRSG.), Europäisches Insolvenzrecht: grundsätzliche Fragestellungen der Prozessrechtsvergleichung, Bielefeld, Gieseking Verlag, 2019.

H. Hess, Die Restrukturierung des Insolvenzrechts: eine Analyse des Richtlinienentwurfs COM(2016) 723 final, Baden-Baden, Nomos, 2019.

T. Himmer, Das europäische Konzerninsolvenzrecht nach der reformierten EuInsVO, Tübingen, Mohr Siebeck, 2019.

J.F. Hoffmann, "Gläubigeranfechtungsklagen und Zuständigkeit nach Maßgabe der EuGVVO: Anmerkung zu EuGH, Urt. v. 4.10.2018, C-337/17, Feniks", GPR, 16, 4, 2019, pp. 168-173.

C.A. Kern/C. Uhlmann, "Internationale Zuständigkeit bei Anfechtungsklagen, die außerhalb eines Insolvenzverfahrens erhoben werden (zu EuGH, 4.10.2018, Rs. C-337/17, Feniks sp. z.o.o. vs. Azteca Products \& Services SL)", IPRax, 39, 6, 2019, pp. 488-493. 
É. KLEIDER, De la faillite internationale à la procédure d'insolvabilité européano-suisse dans le cadre du règlement No 2015/848: les effets en Suisse, Paris, LGDJ, Basel, Helbing Lichtenhahn Verlag, 2019.

C. Koller/E. Lovrek/M. Spitzer, IO: Insolvenzordnung: Mit EuInsVO und EKEG: Kommentar, Wien, Verlag Österreich, 2019.

L. KorHERR, Funktional-teleologische Qualifikation und Gläubigerschutz: eine Untersuchung zur Anwendbarkeit insolvenznaher Gläubigerschutzinstrumente auf EU-Auslandsgesellschaften mit inländischem Verwaltungssitz, München, C.H. Beck, 2019.

T. Kostoula, "Cross-border insolvency of groups of companies under the Regulation (EU) 2015/848", European Company Law, 16, 3, 2019, pp. 74-82.

L. Luchetti/G. Palermo, "Recenti sviluppi normativi in materia di riconoscimento di decreti di fallimento e di concordati stranieri in Svizzera", Diritto del commercio internazionale, 33.2, 2019, pp. 291-307.

T. LuTZI, “Feniks' aus der Asche: internationale Zuständigkeit für die actio pauliana nach der EuGVVO”, $R I W, 65,5,2019$, pp. 252-258.

R. Mangano, "The puzzle of the new European COMI rules: rethinking COMI in the age multinational, digital and glocal enterprises", EBOR, 20, 4, 2019, pp. 779-800.

E. MoustaIRA, International Insolvency Law: National Laws and International Texts, Cham, Springer International Publishing, 2019.

J.A. MüLLER, Internationale Verweisungen und forum (non) conveniens im europäischen Konzerninsolvenzrecht, Berlin, Peter Lang, 2019.

J. PARZINGER, "Der Vorrang für neues Geld nach der Restrukturierungsrichtlinie", ZIP, 40, 37, 2019, pp. 1748-1759.

M. PoESEN, "Once more unto the breach: the actio pauliana is a matter relating to a contract in EU private international law", European Review of Contract Law, 15, 1, 2019, pp. 58-65.

A. Schmidt/B. KNof, EuInsVO: Kommentar, Köln, Heymanns, 2019.

L. SснміDт, Die Zusicherung nach Art. 36 EuInsVO: zugleich ein Beitrag zur Bewältigung grenzüberschreitender Konzerninsolvenzen, Tübingen, Mohr Siebeck, 2019.

I. SCHNEIDER, Registrierte Gegenstände im grenzüberschreitenden Insolvenzverfahren nach der EuInsVO, Baden-Baden, Nomos Verlagsgesellschaft, 2019.

C.H. SeibT/A. von Treuenfeld, "Gesellschafts- und kapitalmarktrechtliche Aspekte der EU-Restrukturierungsrichtlinie", $D B, 72,21,2019$, pp. 1190-1199.

D. SkaURADSZun, "Die Restrukturierungsrichtlinie und das 'verschwitzte' internationale Zivilverfahrensrecht", ZIP, 40, 32, 2019, pp. 1501-1507.

A. Tenenbaum, "Action paulienne et détachement des travailleurs: nouveautés jurisprudentielle et législative: CJUE, 4 oct. 2018, no. C-337/17, Feniks sp. z o.o. c/ Azteca Products \& Services SL”, Revue des contrats, 1, 2019, pp. 154-158.

C. Thole, "Die Abgrenzung zwischen EuInsVO und EuGVVO bei Haftungsklagen gegen Dritte wegen eines Gläubigergesamtschadens (zu EuGH, 6.2.2019, Rs. C-535/17, NK, Konkursverwalter der PI Gerechtsdeurwaarderskantoor BV und von PI vs. BNP Paribas Fortis NV)", IPRax, 39, 6, 2019, pp. $483-488$.

\section{Libre circulación de decisiones en el ámbito patrimonial}

N. BRÜGGEMAnN, Die Anerkennung prorogationswidriger Urteile im europäischen und US-amerikanischen Zivilprozessrecht, Tübingen, Mohr Siebeck, 2019.

D. FouSSARD, "Délai d'exécution d'une ordonnance de saisie conservatoire émise par un État membre et revêtue du caractère exécutoire sur le territoire d'un autre État membre", RCDIP, 2019, pp. 160176.

A. GoŁĄ, "Cross-border implications of fictitious service and unreasoned judgements in the EU (annotation to the German Federal Court of Justice's decision of 10/9/2015, IX ZB 39/13, see below p. 66, no. 7)", IPRax, 39, 1, 2019, pp. 30-35. 
J. von Hein/T. ImM, "Grenzüberschreitende Durchsetzung von Forderungen in der EU: vom Flickenteppich zum System", IWRZ, 4, 3, 2019, pp. 112-118.

M. Ho-DAC, "Précisions sur l'office du juge (d'origine) en matière d'exécution internationale des décisions dans les litiges européens de consommation: CJUE, 4 septembre 2019, Salvoni, C-347/18, ECLI:EU:C:2019:661”, Revue des affaires européennes, 24, 3, 2019, pp. 653-662.

G. Mäsch/M.C. PeIfFER, "Das neue Vollstreckungsregime unter der Brüssel Ia-VO: was bringt der Paradigmenwechsel für den Vollstreckungsgläubiger?", $R I W, 65,5,2019$, pp. 245-252.

H. Muir-WatT, "Sanctionner ou circuler?: les conséquences sur le terrain des effets des jugements de la méconnaissance par le juge second saisi des règles relatives à la litispendance", $R C D I P, 2019$, pp. 487-503.

P. SCHWARZ, Enforcement Shopping im europäischen Rechtsraum: unter besonderer Berücksichtigung des deutschen und polnischen Zwangsvollstreckungsrechts, Jena, Jenaer Wissenschaftliche Verlagsgesellschaft, 2019.

B. UlRICI, "Vollstreckung nach der Brüssel Ia-VO bei Abhängigkeit von einer Sicherheitsleistung", RIW, 65, 7, 2019, pp. 406-414.

L. Volkhausen, "Die Abschaffung des Exequaturverfahrens: ein Weg zu mehr einverstänlichem Vorgehen im europäischen Verfahrensrecht?", en The EU and the matter of concerted acting, 2019, pp. 67-91.

C. Voss, Die Durchbrechung der Rechtskraft nationaler Zivilgerichtsurteile zu Gunsten des unionsrechtlichen "effet utile"?: die Reichweite der "Klausner Holz"-Entscheidung des Europäischen Gerichtshofs und ihre Auswirkungen auf das deutsche Zivilprozessrecht, Baden-Baden, Nomos, 2019.

J. WolBER, "Die Pfändbarkeit eines Gesellschaftsanteils an einer britischen LLP im Inland: zugleich ein Beitrag zu den Grundlagen des europäischen Zwangsvollstreckungsrechts", EuZW, 30, 20, 2019, pp. 863-868.

H. ZIMMER, Die Abschaffung des Exequaturverfahrens im Rahmen der Brüssel Ia-VO, Remscheid, Gardez! Verlag, 2019.

\section{Arbitraje privado internacional}

F. DE ABReu DuARTE, “'But the last word is ours': the monopoly of jurisdiction of the Court of Justice of the European Union in light of the investment cour system", European Journal of International Law, 30, 4, 2019, pp. 1187-1220.

S. AmpatZI, Das AGB-Recht in der nationalen und internationalen Schiedsgerichtsbarkeit im unternehmerischen Geschäftsverkehr: eine Untersuchung im deutschen und griechischen Recht, BadenBaden, Nomos, 2019.

I. Amro, Online arbitration in theory and in practice: a comparative study of cross-border commercial transactions in common law and civil law countries, Newcastle upon Tyne, Cambridge Scholars Publishing, 2019.

A. ANDOLFI, "L'arbitrato in materia di investimenti alla prova dell'effettività del diritto dell'Unione", Rivista dell'arbitrato, XXIX, 1, 2019, pp. 136-159.

A. ANDOLFI, "Il difficile equilibrio tra primauté del diritto dell'Unione e autonomia dell'arbitrato: riflessioni all'indomani della sentenza Achmea", Rivista trimestrale di diritto e procedura civile, LXXIII, 3, 2019, pp. 987-1012.

A. ANDolfi, "L'incerta sorte dell'investment arbitration: note a margine del parere 1/17 della Corte di giustizia", Diritto del commercio internazionale, 33.4, 2019, pp. 755-783.

S. BATTINI, "La grande avventura della giustizia amministrativa, oltre lo Stato: il caso dell'arbitrato internazionale in materia di investimenti", Rivista trimestrale di diritto pubblico, 69, 4, 2019, pp. 1003-1042.

S. BERTOLINI, Die Durchsetzung von ISDS-Entscheidungen in Deutschland: unter besonderer Berücksichtigung aktueller Entwicklungen in der EU, Berlin, Duncker \& Humblot, 2019.

M. Bнатті, Islamic law and international commercial arbitration: The application of shari a in contemporary international commercial arbitration, London, New York, Routledge, 2019. 
P. BINDER, International commercial arbitration and mediation in UNCITRAL model law jurisdictions, $4^{\mathrm{a}}$ ed., Alphen aan den Rijn, Kluwer Law International, 2019.

G. BlankE, "The European Commission as amicus curiae in EU competition arbitration: towards a structured approach", European Competition Law Review, 40, 9, 2019, pp. 438-444.

W. Buchwitz, Schiedsverfahrensrecht, Berlin, Springer, 2019.

B. CENTNER, Iura novit curia in internationalen Schiedsverfahren: eine historisch-rechtsvergleichende Studie zu den Grundlagen der Rechtsermittlung, Tübingen, Mohr Siebeck, 2019.

G. CoOp/G. ShARMA, "Procedural innovations to ISDS in recent trade and investment treaties: a comparison of the USMCA and CETA", Austrian Yearbook on International Arbitration, 2019, pp. 467-492.

J.F. DeliLE, "L'avis 1/17, ou, Le retour en grâce des juridictions internationales auprès de la Cour de justice de l'Union européenne", Revue des affaires européennes, 24, 2, 2019, pp. 347-370.

C. De Stefano, "Spama incentivi, aiuti di Stato e legittimo affidamento: una riflessione sugli arbitrati internazionali d'investimento sulle energie rinnovabili", Diritto del commercio internazionale, 33.2, 2019, pp. 406-416.

F. Emanuele/M. Molfa/R. Monico, "The impact of Brexit on international arbitration", RDIPP, LV, 4, 2019, pp. 856-877.

K. FACH Gómez/A.M. López Rodríguez (Eds.), 60 Years of the New York Convention: key issues and future challenges, Alphen aan den Rijn, Kluwer Law International, 2019.

M. FANOU, "Intra-European Union investor-State arbitration post-Achmea: RIP?: an assessment in the aftermath of the Court of Justice of the European Union, case C-284/16, Achmea, judgment of 6 March 2018, EU:C:2018:158", Maastricht Journal of European and Comparative Law, 26, 2 , 2019, pp. 316-340.

F. Ferrari/S. KRöll (Eds.), Conflict of laws in international commercial arbitration, Huntington, NY, Juris, 2019.

F. FerRari/F. Rosenfeld (EDs.), Inherent powers of arbitrators, Huntington, Juris, 2019.

R.H. Folsom, Principles of international litigation and arbitration, $2^{\mathrm{a}}$ ed., St. Paul, MN, West Academic Publishing, 2019.

S. GösSLING, Europäisches Kollisionsrecht und internationale Schiedsgerichtsbarkeit: die Bedeutung der Rom I-Verordnung bei der Bestimmung des anwendbaren materiellen Rechts durch internationale Handelsschiedsgerichte mit Sitz in der EU, Tübingen, Mohr Siebeck, 2019.

M. Gómez JENE, International commercial arbitration in Spain, Alphen aan den Rijn, Wolters Kluwer, 2019.

F. HeRrmanN/M. JAKOB, "Arbitration cartel damage claims in the EU: still possible after the CDC judgment?: an analysis on the arbitrability of cartel damage claims and the interpretation of the scope of arbitration agreements", Global Competition Litigation Review, 12, 3, 2019, pp. 118-129.

F. IORIO, "Opinion 1/17: has the EU made peace with investment arbitration?", Revue de droit des affaires internationales, $\mathrm{n}^{\circ}$ 4, 2019, pp. 407-420.

R. Khodykin/C. Mulcahy/N. Fletcher, A guide to the IBA Rules on the taking of evidence in international arbitration, Oxford, Oxford University Press, 2019.

G.E. Kodek, "Commercial arbitration after Achmea: business as usual?", en Privatizing dispute resolution: trends and limits, Baden-Baden, Nomos, 2019, pp. 187-208.

M. LaAzouzi, "L'éviction du for de l'action directe de la victime au profit de l'arbitrage international prévu par le contrat d'assurance?", Revue des contrats, 4, 2019, pp. 69-73.

Y. LEVASHOVA, The right of States to regulate in international investment law: the search for balance between public interest and fair and equitable treatment, Alphen aan den Rijn, Kluwer Law International, 2019.

A. Linares CANTillo, El Derecho aplicable en el arbitraje de inversión: la tensión con el Derecho interno, Bogotá, Universidad Externado de Colombia, 2019.

A.M. LóPez Rodríguez/K. FACH Gómez (EDs.), Reconocimiento y ejecución de sentencias arbitrales extranjeras en España y Latinoamérica, Valencia, Tirant lo Blanch, 2019. 
P. Marcisz/A. Orzet-JAKuBowSKA, "The right to be unheard: recognition and enforcement of anti-suit injunctions issued by arbitrations in the EU", Journal of International Dispute Settlement, 10, 1, 2019, pp. 1-22.

D. Murray/W. Antoon, "International arbitration in 2029 in a post-Brexit UK: 10 predictions (and a review of relevant English Court decisions of the last 6 months)", Diritto del commercio internazionale, 33.2, 2019, pp. 309-322.

D. Nigmatullina, Combining mediation and arbitration in international commercial dispute resolution, London, New York, Routledge, 2019.

N.D. O'Malley, Rules of evidence in international arbitration: an annotated guide, $2^{\mathrm{a}}$ ed., London, Informa Law from Routledge, 2019.

M. PIKA, Third-party effects of arbitral awards: res judicata against privies, non-mutual preclusion and factual effects, Alphen aan den Rijn, Kluwer Law International, 2019.

E. Reymond-Eniaeva, Towards a Uniform Approach to Confidentiality of International Commercial Arbitration, Cham, Springer, 2019.

J. RisSE, "The future of arbitration: a poet's prophecy", Journal of International Arbitration, 36, 6, 2019 , pp. 679-692.

B. Sabahi/N. Rubins/D. Wallace, Jr., Investor-State Arbitration, $2^{\mathrm{a}}$ ed., Oxford, Oxford University Press, 2019.

H.-C. Salger/R. Trittmann (Hrsg.), Internationale Schiedsverfahren: Praxishandbuch, München, C.H. Beck, 2019.

H. SAUER, "Europarechtliche Schranken internationaler Gerichte: eine Rekonstruktion der Autonomie des Unionsrechts anlässlich des CETA-Gutachtens des EuGH", JZ, 74, 19, 2019, pp. 925-935.

C. Tiтi/K. FАCH Gómez (ED.), Mediation in international commercial and investment disputes, Oxford, Oxford University Press, 2019.

A. Trakic/J. Benson/P.K. Ahmed (Eds.), Dispute resolution in Islamic finance: alternatives to litigation?, London, New York, Routledge, 2019.

T. VÁRAdy/J.J. Barceló III/S. KRÖLL/A.T. von Mehren, International commercial arbitration: a transnational perspective, $7^{\mathrm{a}}$ ed., St. Paul, MN, West Academic Publishing, 2019.

B.A. WARWAS, "Access to privatized consumer justice: arbitration, ADR, and the future of value-oriented justice in the EU", en Privatizing dispute resolution: trends and limits, Baden-Baden, Nomos, 2019, pp. 325-347.

I. WelSER/A.K. Wolf-Posch, "Private enforcement of cartel infringements before arbitral tribunals", Austrian Yearbook on International Arbitration, 2019, pp. 39-65.

R. Wolff (ED.), New York Convention: Convention on the Recognition and Enforcement of Foreign Arbitral Awards of 10 June 1958: article-by-article commentary, $2^{\mathrm{a}}$ ed., München, Beck, Oxford, Hart Publishing, Baden-Baden, Nomos, 2019.

G. Zeiler/A. Siwy (Eds.), The European Convention on International Commercial Arbitration: a Commentary, Alphen aan den Rijn, Kluwer Law International, 2019.

\section{Derecho de familia internacional}

M. ANDrae, Internationales Familienrecht, $4^{\mathrm{a}}$ ed., Baden-Baden, Nomos, 2019.

K. Boele-Woelki/D. Martiny, Plurality and diversity of family relations in Europe, Cambridge, Intersentia, 2019.

A. DutTA, Familie und Personenstand: ein Handbuch zum deutschen und internationalen Privatrecht, $3^{\mathrm{a}}$ ed., Frankfurt am Main, Berlin, Verlag für Standesamtswesen, 2019.

E. Gitschthaler (HRSG.), Internationales Familienrecht: EU-Verordnungen und Haager Übereinkommen zum Ehe-, Partner- und Kindschaftsrecht: Kommentar, Wien, Verlag Österreich, 2019.

C. JASPERS, "Mandatory mediation from a European and comparative law perspective", en Plurality and diversity of family relations in Europe, 2019, pp. 341-369. 
C. KoHLer, "Anmerkungen zur Parteiautonomie im internationalen Familien- und Erbrecht", en Die Person im internationalen Privatrecht: Liber Amicorum Erik Jayme, Tübingen, Mohr Siebeck, 2019, pp. 9-25.

C. Kohler/W. Pintens, "Entwicklungen im europäischen Personen-, Familien- und Erbrecht 2018-2019”, FamRZ, 66, 18, 2019, pp. 1477-1488.

R. MAGNus, "Die konkludente Rechtswahl im internationalen Erb- und Familienrecht", IPRax, 39, 1, 2019, pp. 8-16.

K. Niethammer-JÜrgens/M. ERb-Klünemann, Internationales Familienrecht in der Praxis: ein Leitfaden, $2^{\mathrm{a}}$ ed., Frankfurt am Main, Wolfgang Metzner Verlag, 2019.

Y. Nishitani, Identité culturelle en droit international privé de la famille, Leiden, Boston, Brill Nijhoff, 2019.

A. SAHNER, Materialisierung der Rechtswahl im internationalen Familienrecht: zur Bedeutung des Schwächerenschutzes im europäischen Kollisionsrecht, Tübingen, Mohr Siebeck, 2019.

M. STREICHER, Familiensachen mit Auslandsberührung: Anwaltshandbuch zur effizienten Bearbeitung familienrechtlicher Mandate mit Auslandsbezug, $3^{\text {a }}$ ed., Köln, Deubner Recht \& Praxis, 2019.

\section{Persona física}

S. Adroher Biosca, La plurinacionalidad en Derecho internacional privado español, Madrid, Reus Editorial, 2019.

M. Gebauer/H.-P. Mansel/G. Schulze (Hrsg.), Die Person im internationalen Privatrecht: Liber Amicorum Erik Jayme, Tübingen, Mohr Siebeck, 2019.

H.-P. Mansel, "Methoden des internationalen Privatrechts: Personalstatut, Verweisung und Anerkennung", en Die Person im internationalen Privatrecht: Liber Amicorum Erik Jayme, Tübingen, Mohr Siebeck, 2019, pp. 27-46.

\section{Matrimonio y parejas de hecho}

E. Díez Peralta, El matrimonio infantil y forzado en el Derecho internacional: un enfoque de género y de derechos humanos, Valencia, Tirant lo Blanch, 2019.

K. DE La Durantaye, "Same same but different?: das IPR der Ehe für alle nach Inkrafttreten des Gesetzes zur Umsetzung des Eheöffnungsgesetzes", IPRax, 39, 4, 2019, pp. 281-290.

F. Pesce, "La nozione di 'matrimonio': diritto internazionale privato e diritto materiale a confronto", $R D I P P$, LV, 4, 2019, pp. 777-818.

C.S. RupP, "Verliebt, verlobt, rückabgewickelt?: Ansprüche bei der Auflösung von Verlöbnissen aus grenzüberschreitender Perspektive", RabelsZ, 83, 1, 2019, pp. 154-187.

M. SHAHID, "How do European courts approach the sensitive topic of same-sex marriage?: an analysis of the case law of the ECtHR and the CJEU in the 'Catch 22'-field of equal marriage rights", European Yearbook on Human Rights 2019, 2019, pp. 237-259.

D. ZANNONI, "Jurisdiction and law applicable to civil partnership under Italian law", ZEuP, 27, 3, 2019, pp. 571-592.

\section{Régimen económico matrimonial y de parejas registradas}

S. Arnold/S. LaImer (HrSG.), Die europäischen Güterrechtsverordnungen: internationales Ehegüterrecht und Güterrecht für LebenspartnerInnen in Europa: Handbuch, Wien, Verlag Österreich, 2019.

N.C. BARREDA, "Entre la lex causae et les lois de police de la lex fori: quelle alternative pour la protection du logement familial dans le règlement 'régimes matrimoniaux'?", European Review of Private Law, 27, 3, 2019, pp. 583-615. 
U. Bergquist/D. Damascelli/R. Frimston/P. Lagarde/B. Reinhartz, The EU regulations on matrimonial and patrimonial property, Oxford, Oxford University Press, 2019.

M. BRosch, Rechtswahl und Gerichtsstandsvereinbarung im internationalen Familien- und Erbrecht der EU, Tübingen, Mohr Siebeck, 2019.

P. BRUNo, I regolamenti europei sui regimi patrimoniali dei coniugi e delle unioni registrate: commento ai regolamenti (UE) 24 giugno 2016, nn. 1103 e 1104 applicabili dal 29 gennaio 2019, Milano, Giuffrè Francis Lefebvre, 2019.

C. DöBEREINER/S. FrANK, Internationales Güterrecht für die Praxis: die neuen EU-Güterrechtsverordnungen, Bielefeld, Verlag Ernst und Werner Gieseking, 2019.

A. DutTA, "Europäische Güterrechtsverordnungen und deutsche Durchführungsgesetzgebung", FamRZ, 66, 17, 2019, pp. 1390-1400.

S. GRÄF, Drittbeziehungen und Drittschutz in den Europäischen Güterrechtsverordnungen, Tübingen, Mohr Siebeck, 2019.

J.L. Iglesias Buigues/G. Palao Moreno (DiR.), Régimen económico matrimonial y efectos patrimoniales de las uniones registradas en la Unión Europea: comentarios a los Reglamentos (UE) n. 2016/1103 y 2016/1104, Valencia, Tirant lo Blanch, 2019.

A. LAS CASAS, "La nozione autonoma di 'regime patrimoniale tra coniugi' del regolamento UE 2016/1103 e i modelli nazionali", Le nuove leggi civili commentate, XLII, 6, 2019, pp. 1529-1555.

S. MARINo, I rapporti patrimoniali della famiglia nella cooperazione giudiziaria civile dell'Unione europea, Milano, Giuffrè Francis Lefebvre, 2019.

P. De PAGe/I. DE Stefani, Régimes conventionnels et droit international privé, Bruxelles, Bruylant, 2019.

G. Ring/L. OlsEn-Ring, "Das Gesetz zum internationalen Güterrecht und zur Änderung von Vorschriften des internationalen Privatrechts", NotBZ, 23, 4, 2019, pp. 124-132.

G. Ring/L. OlsEn-Ring, "Das neue dänische IPR zum Ehegüterrecht”, IPRax, 39, 4, 2019, pp. 347-353.

C. Rudolf, "European Property Regimes Regulations: choice of law and the applicable law in the absence of choice by the parties", LeXonomica, 11, 2, 2019, pp. 127-150.

R. Schuz, "Choice of law in relation to matrimonial property in the 21 st century", Journal of Private International Law, 15, 1, 2019, pp. 1-49.

M. SonNEnTAG, "Die erbrechtliche Qualifikation des güterrechtlichen Viertels durch den EuGH und ihre Konsequenzen: zugleich Besprechung von EuGH, Urteil v. 1.3.2018, C-558/16 (Mahnkopf)", JZ, 74, 13, 2019, pp. 657-666.

M. Uiтz, "Kontroversen im europäischen Güterkollisionsrecht", ZfRV, 60, 5, 2019, pp. 213-229.

A.L. VALVO, "Integrazione europea in cammino: i regolamenti europei in materia di regimi patrimoniali tra coniugi e in materia di effetti patrimoniali delle unioni registrate", Rivista della cooperazione giuridica internazionale, XXII, 61, 2019, pp. 11-17.

M. Wendland, "Verfahrensrechtliche Probleme im System internationaler Entscheidungszuständigkeit der neuen europäischen Güterrechtsverordnungen: Altbekanntes und Innovatives aus dem Experimentierlabor der Europäischen Kommission", IPRax, 39, 1, 2019, pp. 1-8.

\section{Crisis matrimoniales}

C. Althammer (ED.), Brussels IIa - Rome III: article-by-article commentary, München, Beck, Oxford, Hart, Baden-Baden, Nomos, 2019.

J. Antomo, "Privatscheidungen und der neue Art. 17 Abs. 2 EGBGB: Rom III-VO à la berlinoise", StAZ, 72, 2, 2019, pp. 33-42.

G. Biagioni, "Il nuovo Regolamento (UE) 2019/1111 relativo alla competenza, al riconoscimento e all'esecuzione delle decisioni in materia matrimoniale e di responsabilità genitoriale, e alla sottrazione internazionale", RDI, CII, 4, 2019, pp. 1169-1178.

M. Brosch, Rechtswahl und Gerichtsstandsvereinbarung im internationalen Familien- und Erbrecht der EU, Tübingen, Mohr Siebeck, 2019.

M. CoRBEtT, "The public law aspects of the Brussels IIbis Regulation through an Irish lens", en Plurality and diversity of family relations in Europe, 2019, pp. 203-224. 
A. DAvì/A. ZAnobetTI, "Il nuovo Regolamento UE 2019/1111 e la circolazione di separazioni e divorzi nello spazio giudiziario europeo", Studi sull 'integrazione europea, XIV, 3, 2019, pp. 749-764.

A. DutTA, "Private divorces outside Rome III and Brussels II bis?: the Sahyouni gap: case C-372/16, Soha Sahyouni v. Raja Mamich, judgment of the Court (First Chamber) of 20 December 2017, EU:C:2017:988", CMLR, 56, 6, 2019, pp. 1661-1672.

M. HaRper/D. GoOdman, International trust and divorce litigation, $3^{\mathrm{a}}$ ed., London, Edinburgh, LexisNexis, 2019.

K. KAESLING, "The recognition of religious private divorces in Europe: from conflict of laws to conflict of cultures?", en Plurality and diversity of family relations in Europe, 2019, pp. 257-284.

L. SchlÜRmann, "Art. 17 Abs. 2 EGBGB n. F. als überschießende Anwendung der Rom III-VO: das neue Kollisionsrecht für Privatscheidungen nach Sahyouni”, FamRZ, 66, 13, 2019, pp. 1035-1040.

O. TAHERI, Die Anwendung des iranischen Scheidungsrechts vor deutschen Gerichten: in Theorie und Praxis, Regensburg, S. Roderer Verlag, 2019.

\section{Filiación natural}

C. BENICKE, "Anpassungsproblematik beim Zusammentreffen deutschen Erbrechts mit englischem Eltern-Kind-Statut (zu OLG München, 10.2.2017, 34 Wx 175/16, unten S. 162, Nr. 16)”, IPRax, 39, 2, 2019, pp. 132-140.

H. HuRPY, "La judiciarisation par défaut du lien de filiation des enfants nés d'une GPA transfrontière avec la mère d'intention", Revue de l'Union européenne, ${ }^{\circ}$ 631, 2019, pp. 486-494.

M.S. Quicios Molina, "Regulación por el ordenamiento español de la gestación por sustitución: dónde estamos y hasta dónde podemos llegar", Revista de Derecho Privado, Enero-febrero (2019), núm. 1, pp. 3-46.

P. Mоsтошікк (ED.), Fundamental legal problems of surrogate motherhood: global perspective, Warszawa, Wydawnictwo Instytutu Wymiaru Sprawiedliwości, 2019.

L. Sales Pallarés, "La pérdida del interés (superior del menor) cuando se nace por gestación subrogada", Cuadernos de Derecho Transnacional, 11, 2, 2019, pp. 326-347.

\section{Adopción internacional}

S. ADROHER BiosCA, "La vuelta de las adopciones simples a España y la comunidad jurídica Iberoamericana", en X Congreso de las Academias Jurídicas de Iberoamérica: Madrid (España) 22, 23 y 24 de noviembre de 2018, vol. 2, 2019, pp. 43-55.

C. AzCÁRraga Monzonís, "La intermediación y los organismos acreditados en las adopciones internacionales. Reflexiones derivadas del nuevo Reglamento de adopción internacional", REEI, nº 38, 2019.

M.T. PÉrez Giménez, "El nuevo régimen jurídico de la adopción internacional", Revista Crítica de Derecho Inmobiliario, $95, \mathrm{n}^{\circ} 772,2019$, pp. 763-805.

M.J. SÁnchez CANO, “Adopción simple: inscripción en el registro civil y adquisición de la nacionalidad española: una cuestión pendiente", en A. Cebrián SALVAt/I. Lorente Martínez (Dir.), Protección de menores y Derecho internacional privado, Granada, Editorial Comares, 2019, pp. 35-60.

M.J. SÁNCHEZ CANO, "Cuestiones vinculadas a la constitución de la adopción de mayores de edad: análisis desde el Derecho internacional privado español", Cuadernos de Derecho Transnacional, 11, 1, 2019, pp. 904-916.

M.J. SÁNChEZ CANO, "El reconocimiento incidental de una adopción internacional vinculado a la adquisición de la nacionalidad española: aspectos prácticos", Cuadernos de Derecho Transnacional, 11, 2, 2019, pp. 801-814.

M.J. SÁnchez Cano/Y. Romero Matute, "Circunstancias que impiden o condicionan la adopción: el alcance de la denominada 'cláusula chadiana", Cuadernos de Derecho Transnacional, 11, 1, 2019, pp. 917-928. 


\section{Protección de menores}

S. Adroher Biosca, "Estancias temporales de menores extranjeros en España: régimen jurídico vigente y desafíos de futuro", Cuadernos de Derecho Transnacional, 11, 1, 2019, pp. 51-62.

C. Althammer (ED.), Brussels IIa - Rome III: article-by-article commentary, München, Beck, Oxford, Hart, Baden-Baden, Nomos, 2019.

I. Barrière-Brousse, "Règlement Bruxelles II bis: CJUE, 1re ch., 16 janv. 2019, aff. C-386/17, Stefano Liberato c/ Luminita Luisa Grigorescu: note", JDI Clunet, 146, 4, 2019, pp. 1235-1242.

G. Biagioni, "Il nuovo Regolamento (UE) 2019/1111 relativo alla competenza, al riconoscimento e all'esecuzione delle decisioni in materia matrimoniale e di responsabilità genitoriale, e alla sottrazione internazionale", RDI, CII, 4, 2019, pp. 1169-1178.

L. CARPANETO, "Impact of the best interests of the child on the Brussels II ter Regulation", en Fundamental rights and best interest of the child in transnational families, 2019, pp. 265-285.

A. Cebrián Salvat/I. Lorente Martínez (Dir.), Protección de menores y Derecho internacional privado, Granada, Editorial Comares, 2019.

C. Chalas, "Raison et sentiments en matière d'enlèvement international d'enfant: quel équilibre dans les jurisprudences de la Cour européenne des droits de l'homme, de la Cour de justice de l'Union européenne de l'Union européenne et de la Cour de cassation?", RCDIP, 2019, pp. 111-126.

M. DEL C. ChÉLIZ INGLÉs, La sustracción internacional de menores y la mediación: retos y vías prácticas de solución, Valencia, Tirant lo Blanch, 2019.

A. Del Ser López/D. Carrizo Aguado, "Reglas de competencia judicial internacional en materia de responsabilidad parental : análisis del foro de la 'residencia habitual del menor' y estudio de la 'competencia residual'”, Revista Aranzadi Unión Europea, XLV, 10, 2019, pp. 49-74.

J. Delgado SÁez, "The right of the child to be heard in parental responsibility proceedings", en Plurality and diversity of family relations in Europe, 2019, pp. 225-236.

S. Fulli-Lemaire, "La prorogation de compétences en matière de responsabilité parentale dans le cadre du règlement Bruxelles II bis", RCDIP, 2019, pp. 457-469

U.P. GRUBER, "Der gewöhnliche Aufenthalt von Säuglingen und Kleinkindern (zu EuGH, 28.6.2018, C-512/17, unten S. 248, Nr. 21)", IPRax, 39, 3, 2019, pp. 217-221.

C. Honorati, "La tutela dei minori migranti e il diritto internazionale privato: quali rapporti tra Dublino III e Bruxelles II-bis?", RDIPP, LV, 4, 2019, pp. 691-713.

R. LAMONT, "Protecting children's rights after child abduction: the interaction of the CJEU and ECtHR in interpreting Brussels II bis", en Fundamental rights and best interest of the child in transnational families, 2019, pp. 225-242.

K. LENAERTS, "Child abduction in the case law of the Court of Justice of the European Union and of the European Court of Human Rights", en Human rights with a human touch: liber amicorum Paul Lemmens, Cambridge, Intersentia, 2019, pp. 313-331.

C. Thomale, "Der gewöhnliche Aufenthalt im europäischen Kindschaftsrecht: Anmerkung zu EuGH 17.10.2018, C-393/18 PPU”, GPR, 16, 4, 2019, pp. 173-176.

\section{Alimentos}

I. BARrière-Brousse, "Règlement Bruxelles II bis: CJUE, 1re ch., 16 janv. 2019, aff. C-386/17, Stefano Liberato c/ Luminita Luisa Grigorescu: note", JDI Clunet, 146, 4, 2019, pp. 1235-1242.

M. Brosch, Rechtswahl und Gerichtsstandsvereinbarung im internationalen Familien- und Erbrecht der EU, Tübingen, Mohr Siebeck, 2019.

N. JouAnT, "La loi applicable à l'action en révision de la pension alimentaire intentée devant les tribunaux de la résidence habituelle du débiteur d'aliments", $R C D I P, 2019$, pp. 146-159.

V. LIPP, "Statut des Kindesunterhalts nach Aufenthaltswechsel (zu EuGH, 7.6.2018, C-83/17, KP vs. LO, unten S. 430, Nr. 41)", IPRax, 39, 5, 2019, pp. 400-405.

L. Rass-Masson, "Protocole de La Haye du 23 novembre 2007: CJUE, 6e ch., 7 juin 2018, aff. C-83/17", JDI Clunet, 146, 3, 2019, pp. 867-878. 


\section{Sucesión mortis causa}

H. Amann, "Fiktion des früheren Erbstatuts unter der Herrschaft der EuErbVO", DNotZ, 118, 5, 2019, pp. 326-345.

C. BeNICKE, "Anpassungsproblematik beim Zusammentreffen deutschen Erbrechts mit englischem Eltern-Kind-Statut (zu OLG München, 10.2.2017, 34 Wx 175/16, unten S. 162, Nr. 16)", IPRax, 39, 2, 2019, pp. 132-140.

M. BRosch, Rechtswahl und Gerichtsstandsvereinbarung im internationalen Familien- und Erbrecht der EU, Tübingen, Mohr Siebeck, 2019.

A.-L. Calvo Caravaca/J. Carrascosa González (Dir.), Litigación Internacional en la Unión Europea (IV): Comentario al Reglamento (UE) núm. 650/2012 del Parlamento Europeo y del Consejo sobre sucesiones mortis causa, Cizur Menor, Navarra, Thomson Reuters Aranzadi, 2019.

K. DorTH, Das Verhältnis von Erbschein und Europäischem Nachlasszeugnis, Baden-Baden, Nomos, Bern, Stämpfli, München, Beck, 2019.

A. Dutta/W. Wurmnest, European private international law and Member State treaties with third States: the case of European succession regulation, Cambridge, Intersentia, 2019.

C. GRIECO, Il ruolo dell'autonomia della volontà nel diritto internazionale privato delle successioni transfrontaliere, Milano, Giuffrè Francis Lefebvre, 2019.

M. HÖRDT, Pflichtteilsrecht und EU-ErbVO, Berlin, Frank \& Timme, Verlag für Wissenschaftliche Literatur, 2019.

C. KoHLER, "Anmerkungen zur Parteiautonomie im internationalen Familien- und Erbrecht”, en Die Person im internationalen Privatrecht: Liber Amicorum Erik Jayme, Tübingen, Mohr Siebeck, 2019, pp. 9-25.

B. KRESSE, "Die Prüfungskompetenz des EuGH in kollisionsrechtlichen Vorabentscheidungsverfahren: erbrechtliche Qualifikation des $§ 1371$ Abs. 1 BGB?", GPR, 16, 4, 2019, pp. 195-200.

T. KRŪMIN̦Š, "Application of the EU Succession Regulation in practice: the case of Latvia and beyond", Journal of Private International Law, 15, 2, 2019, pp. 365-392.

L. Kunz, "Entscheidungen in Erbsachen: zur Rolle der freiwilligen Gerichtsbarkeit in der EuErbVO aus Sicht des deutschen Erbverfahrensrechts", GPR, 16, 1, 2019, pp. 34-42.

S. KURTH, "Wechselleben zwischen der spanischen Costa Brava und der ostwestfälischen Provinz: Ermittlung des gewöhnlichen Aufenthalts eines Erblassers, der sich in zwei Staaten abwechselnd aufhält (zu OLG Hamm, 2.1.2018, 10 W 35/17, unten S. 151, Nr. 11)", IPRax, 39, 2, 2019, pp. 123-127.

R. Magnus, "Die konkludente Rechtswahl im internationalen Erb- und Familienrecht", IPRax, 39, 1, 2019, pp. 8-16.

C.F. NoRDMEIER, "Die Aufnahme einzelner Nachlassgegenstände in das Europäische Nachlasszeugnis: zum durch den Todesfall bedingten Rechtserwerb und zur Reichweite der Art. 68 lit. 1 und $\mathrm{m}$ EuErbVO (zu OLG Nürnberg, 5.4.2017, 15 W 299/17, unten S. 327, Nr. 31, OLG Nürnberg, 25.4.2017, 15 W 318/17, unten S. 328, Nr. 32 und OLG Nürnberg, 27.10.2017, 15 W 1461/17, unten S. 328, Nr. 33)", IPRax, 39, 4, 2019, pp. 306-312.

V.T. Profensner, Disposition im internationalen Erbrecht: Rechtswahl und parteiautonome Zuständigkeitsbestimmung nach der Europäischen Erbrechtsverordnung unter besonderer Berücksichtigung der beteiligten Interessen, Berlin, Peter Lang, 2019.

T. Puig Stoltenberg, Die Parteiautonomie im europäischen Erbrecht, Frankfurt am Main, Peter Lang, 2019.

C. StrehlKe-VerkÜHLEn, Der Erbverzicht: eine rechtsvergleichende und kollisionsrechtliche Betrachtung, Jena, Jenaer Wissenschaftliche Verlagsgesellschaft, 2019.

R. Süss, Erbrecht in Europa, Bonn, Zerb, 2019.

V.T. Profensner, Disposition im internationalen Erbrecht: Rechtswahl und parteiautonome Zuständigkeitsbestimmung nach der Europäischen Erbrechtsverordnung unter besonderer Berücksichtigung der beteiligten Interessen, Berlin, Lang, 2019.

M. SonNentaG, "Die erbrechtliche Qualifikation des güterrechtlichen Viertels durch den EuGH und ihre Konsequenzen: zugleich Besprechung von EuGH, Urteil v. 1.3.2018, C-558/16 (Mahnkopf)", JZ, 74, 13, 2019, pp. 657-666. 
K. Thorn/C. Lasthaus, "Rechtsnachfolge in Immobilien unter der Europäischen Erbrechtsverordnung (zu EuGH, 12.10.2017, Rs. C-218/16, Kubicka, unten S. 58, Nr. 4, und OGH, 29.8.2017, 5 Ob 108/17v, unten S. 62, Nr. 5 sowie OGH, 21.12.2017, 5 Ob 186/17i, unten S. 64, Nr. 6)", IPRax, 39, 1, 2019, pp. 24-30.

F.C. VILLATA, "Predictability first!: fraus legis, overriding mandatory rules and ordre public under EU regulation 650/2012 on succession matters", RDIPP, LV, 4, 2019, pp. 714-738.

W. WuRMNEST/B. WöSSNER, "Kollisionsrechtliche Staatsverträge mit Drittstaaten in Europa: ein Blick auf die 'Achillesferse' der EuErbVO”, ZVglRWiss, 118, 4, 2019, pp. 449-483. 